\title{
Polyakov loop effects on the phase diagram in strong-coupling lattice QCD
}

\section{$\operatorname{AUTHOR}(\mathrm{S})$ :}

Miura, Kohtaroh; Kawamoto, Noboru; Nakano, Takashi Z.; Ohnishi, Akira

\section{CITATION:}

Miura, Kohtaroh ...[et al]. Polyakov loop effects on the phase diagram in strong-coupling lattice QCD. Physical Review D 2017, 95(11)

ISSUE DATE:

2017-06-01

URL:

http://hdl.handle.net/2433/233202

RIGHT:

(C) 2017 American Physical Society 
PHYSICAL REVIEW D 95, 114505 (2017)

\title{
Polyakov loop effects on the phase diagram in strong-coupling lattice QCD
}

\author{
Kohtaroh Miura, ${ }^{1,2, *}$ Noboru Kawamoto, ${ }^{3}$ Takashi Z. Nakano, ${ }^{4}$ and Akira Ohnishi ${ }^{5}$ \\ ${ }^{1}$ Centre de Physique Theorique(CPT), Aix-Marseille University, Campus de Luminy, \\ Case 907, 163 Avenue de Luminy, 13288 Marseille cedex 9, France \\ ${ }^{2}$ Kobayashi-Maskawa Institute for the Origin of Particles and the Universe, Nagoya University, \\ Nagoya 464-8602, Japan \\ ${ }^{3}$ Department of Physics, Faculty of Science, Hokkaido University, Sapporo 060-0810, Japan \\ ${ }^{4}$ KOZO KEIKAKU ENGINEERING Inc., Tokyo 164-0012, Japan \\ ${ }^{5}$ Yukawa Institute for Theoretical Physics, Kyoto University, Kyoto 606-8502, Japan
}

(Received 28 October 2016; published 15 June 2017)

\begin{abstract}
We investigate the Polyakov loop effects on the QCD phase diagram by using the strong-coupling $\left(1 / g^{2}\right)$ expansion of the lattice QCD (SC-LQCD) with one species of unrooted staggered quark, including $\mathcal{O}\left(1 / g^{4}\right)$ effects. We take account of the effects of Polyakov loop fluctuations in Weiss mean-field approximation (MFA), and compare the results with those in the Haar-measure MFA (no fluctuation from the mean-field). The Polyakov loops strongly suppress the chiral transition temperature in the secondorder/crossover region at small chemical potential $(\mu)$, while they give a minor modification of the firstorder phase boundary at larger $\mu$. The Polyakov loops also account for a drastic increase of the interaction measure near the chiral phase transition. The chiral and Polyakov loop susceptibilities $\left(\chi_{\sigma}, \chi_{\ell}\right)$ have their peaks close to each other in the second-order/crossover region. In particular in Weiss MFA, there is no indication of the separated deconfinement transition boundary from the chiral phase boundary at any $\mu$. We discuss the interplay between the chiral and deconfinement dynamics via the bare quark mass dependence of susceptibilities $\chi_{\sigma, \ell}$.
\end{abstract}

DOI: 10.1103/PhysRevD.95.114505

\section{INTRODUCTION}

The phase diagram of quantum chromodynamics (QCD) at finite temperature $(T)$ and/or quark chemical potential $(\mu)$ $[1,2]$ provides a deep insight into the Universe. At the few microseconds after the big-bang, a quark-gluon plasma (QGP) is supposed to undergo the QCD phase transition/ crossover, which results in confinement of color degrees of freedom and the dynamical mass generation of hadrons. In fact, the first principle calculations based on lattice QCD Monte Carlo simulations (LQCD-MC) indicates the crossover around $T_{c}=145-195(\mathrm{MeV})$ [3]. In compact star cores, a cold-dense system would appear, where various interesting phases are expected [4-8].

The QCD phase transition can be investigated in the laboratory experiments [9]: Circumstantial experimental evidence at the Relativistic Heavy-Ion Collider (RHIC) in Brookhaven National Laboratory together with theoretical arguments implies that the QGP is created in heavy-ion collisions at $\sqrt{s_{N N}}=200 \mathrm{GeV}$, and recent experiments at the Large Hadron Collider (LHC) in CERN give stronger evidence. Probing the phase diagram at finite $\mu$, in particular the critical point $(C P)$ [10], is a central topic in the ongoing and future heavy-ion collision experiments at the Facility for Antiproton and Ion Research (FAIR) at GSI, the Nuclotron-based Ion Collider fAcility (NICA) at

*kohtaroh.miura@cpt.univ-mrs.fr; miura@kmi.nagoya-u.ac.jp
JINR, and the beam energy scan program at RHIC [11]. Unfortunately, the first principle studies by LQCD-MC loses the robustness at finite $\mu$ due to the notorious sign problem [1,12-15]. Many interesting subjects, for example, the location of $C P$, the equation of state (EOS) at high density, are still under debate.

The QCD phase diagram may be characterized by two underlying dynamics, the chiral and deconfinement transitions, which are associated with the spontaneous breaking of the chiral symmetry in the chiral limit and the $Z_{N_{c}}$ center symmetry of the color $\mathrm{SU}\left(N_{c}\right)$ gauge group in the heavy quark mass limit, respectively. The order parameter is the chiral condensate $(\sigma) /$ Polyakov loop $(\ell)$ for the chiral/ deconfinement transition. Although the $Z_{N_{c}}$ symmetry is explicitly broken by the quark sector (with a finite or vanishing mass), the Polyakov loops are still important degrees of freedom to be responsible for the thermal excitation of quarks near the chiral phase transition. The interplay between the $\sigma$ and $\ell$ is under active scrutiny; the LQCD-MC reports that the chiral and Polyakov loop susceptibilities show their peaks at almost the same temperatures for $\mu=0$, and the separation of two dynamics is proposed at finite $\mu$ in several models [2].

We investigate the QCD phase diagram by using the strong-coupling expansion in the lattice QCD (SC-LQCD), which provides a lattice-based and well-suited framework for the chiral and deconfinement transitions without a serious contamination by the sign problem. The SCLQCD has been successful since the beginning of the 
lattice gauge theory [16-19], and revisited after the QGP discovery at RHIC as an instructive guide to the QCD phase diagram [20-33]. It is remarkable that a promising phase diagram structure has been obtained even in the strong-coupling limit $\left(\beta=2 N_{c} / g^{2} \rightarrow \infty\right)$ with mean-field approximation (MFA) $[20,22,24]$, and exactly determined based on the Monomer-Dimer-Polymer (MDP) formulation [30] and the Auxiliary Field Monte Carlo simulation [33]. The MFA results are then shown to be capturing the essential feature of the exact phase diagram.

In Fig. 1, we summarize the SC-LQCD studies on the color SU(3) QCD phase diagram using MFA. Based on the success in the strong-coupling limit (top in the second column), we have investigated the phase diagram [25,27] by taking account of the next-to-leading order (NLO, $\mathcal{O}\left(1 / g^{2}\right)$, middle in the second column) and the next-tonext-to-leading order $\left(\mathrm{NNLO}, \mathcal{O}\left(1 / g^{4}\right)\right.$, bottom in the second column) of the strong-coupling expansion. The chiral phase transition temperature $T_{c}$ is strongly suppressed by the NLO effects, and the phase diagram evolves into the empirical shape with increasing lattice coupling $\beta=2 N_{c} / g^{2}$, while the NNLO effects give much milder corrections.

In the works mentioned above (listed in the second column of Fig. 1), the main focus was put on the chiral dynamics, rather than the $Z_{N_{c}}$ deconfinement dynamics, which is another important dynamics described by the Polyakov loops $\ell$ of the pure-gluonic sector. The SC-LQCD has been well-suited to include both dynamics at the strong-coupling limit [34-36] (top in third and fourth columns in Fig. 1); the strong-coupling limit for the quark sector is combined with the leading-order effect of the Polyakov loops in the pure-gluonic sector and the quark determinant term provides the lattice-based derivation of the $\sigma-\ell$ coupling. It is intriguing to include the higher-order of the strong-coupling expansion, which has been carried out in our previous work [28] (middle and bottom lines in third and last columns in Fig. 1); we have shown that the Polyakov loop effects combined with finite lattice couplings $\beta$ further suppresses the chiral transition temperature $T_{c}$, which reproduces the results of LQCD-MC simulations
[37-39] at $\mu=0$ in the certain lattice coupling range $\beta \sim 4$. Thus, the long-standing problem of the SC-LQCD-too large $T_{c}$-is greatly relaxed by the Polyakov loops. Moreover, the Polyakov loop sector at the chiral phase transition $\sim \mathcal{O}\left(\left[1 / g^{2}\right]^{1 / T_{c}}\right)$ is found to be comparable with the quark sector with NLO $\left[\mathcal{O}\left(1 / g^{2}\right)\right]$ and NNLO $\left[\mathcal{O}\left(1 / g^{4}\right)\right]$ at $T_{c}(\beta \sim 4) \sim 0.5-0.6$ (in lattice units); the Polyakov loop effects are necessary to evaluate $T_{c}$ with respect to the order counting of the strong-coupling expansion.

In our previous paper [28], however, the analysis was limited at vanishing chemical potential $\mu=0$, while the finite $\mu$ region receives a growing interest by the forthcoming experiments focusing the $C P$ and high density phase. The purpose of the present paper is to extend our previous work [28] to the finite $\mu$ region, and to investigate the Polyakov loop effects on the whole region of the QCD phase diagram as indicated by red-solid characters in Fig. 1. We adopt two approximation schemes for the Polyakov loops, a simple mean-field treatment (Haar-measure MFA) and an improved treatment with fluctuation effects (Weiss MFA). Through the various comparisons indicated by the arrows in Fig. 1, we elucidate the effect of the Polyakov loop itself, either the effects of the Polyakov loop fluctuations, as well as the higher-order (NNLO) effects of the strong-coupling expansion. In particular, we focus on thermodynamic quantities, which is of great interest in the study of the equation of state for quark matter but has been challenging in SC-LQCD. Moreover, we discuss the interplay between the chiral and deconfinement dynamics at finite $\mu$ via the bare quark mass dependence of susceptibilities $\chi_{\sigma, \ell}$.

We employ one species (unrooted) of staggered fermion, which has a $U_{\chi}(1)$ chiral symmetry in the strong-coupling region and becomes the four flavor QCD with degenerate masses in the continuum limit. We investigate the $U_{\chi}(1)$ chiral phase transition/crossover at finite $T$ and $\mu$ in color $\mathrm{SU}\left(N_{c}=3\right)$ gauge group in the $3+1$ dimension $(d=3)$. Our focus is not necessarily put on quantitative prediction of the realistic phase diagram, but we attempt to clarify

\begin{tabular}{|c|c|c|c|}
\hline Quark Sec. & w.o. PLoop & w. PLoop, Weiss MFA. & w. PLoop, Haar-Meas. MFA. \\
\hline $\begin{array}{l}\text { LO. } \\
\left(1 / g^{0}\right)\end{array}$ & $\begin{array}{l}\text { Damgaard et al. [42] } \\
\text { Fukushima [20] } \\
\text { Nishida [22] } \\
\text { Kawamoto et al. [24] }\end{array}$ & Fukushima [36] & $\begin{array}{l}\text { Gocksch et al. [34] } \\
\text { Ilgenfritz et al. [35] } \\
\text { Fukushima [36] }\end{array}$ \\
\hline $\begin{array}{l}\text { NLO. } \\
\left(1 / g^{2}\right)\end{array}$ & $\begin{array}{r}\text { Miura et al. }[25] \\
\text { Study of } \\
\text { PLoop E }\end{array}$ & $\begin{array}{l}\mu=0 \text { : Nakano et al. }[28] \\
\mu>=0 \text { : Miura et al. } \\
\quad \text { (Present Work) }\end{array}$ & $\begin{array}{l}\mu=0 \text { : Nakano et al }[28] \\
\mu>=0 \text { : Miura et al. } \\
\text { (Present Work) Study of }\end{array}$ \\
\hline $\begin{array}{l}\text { NNLO. } \\
\left(1 / g^{4}\right)\end{array}$ & Nakano et al. [27] & $\mu=0$ : Nakano et al. [28] & $\begin{array}{l}\mu=0 \text { : Nakano et al. }[28] \text { Effects } \\
\mu>=0 \text { : Miura et al. } \\
\text { (Present Work) }\end{array}$ \\
\hline
\end{tabular}

FIG. 1. The summary of the SC-LQCD studies on the QCD phase diagram for color SU(3) using MFA. 
which effects make the SC-LQCD phase diagram being closer to realistic one. Such lattice based arguments would be instructive to future LQCD-MC studies on the QCD phase diagram, even though the flavor-chiral structure in the present study is different from the real-life QCD with $2+1$ flavors.

This paper is organized as follows: In Sec. II, we explain the effective potential in strong-coupling lattice QCD with Polyakov loop effects. In Sec. III, we investigate the phase diagram and related quantities by using the effective potential. In Sec. IV, we summarize our work and give a future perspective. The Appendix is devoted to the review of the effective potential derivation.

\section{STRONG-COUPLING LATTICE QCD WITH POLYAKOV LOOP EFFECTS}

We explain the effective potential of the strong-coupling lattice QCD including the Polyakov loop effects. The derivation has been detailed in our previous work [28], and recapitulated in the Appendix in this paper. Here we explain the essential property of the effective potential. We will work on lattice units $a=1$ in color $\mathrm{SU}\left(N_{c}=3\right)$ gauge and $3+1$ dimension $(d=3)$. The parameters in the effective potential are the lattice coupling $\beta=2 N_{c} / g^{2}$, lattice bare quark mass $m_{0}$, lattice temperature $T=1 / N_{t}$ $\left(N_{t}=\right.$ temporal lattice extension), and quark chemical potential $\mu$.

The effective potential $\mathcal{F}_{\text {eff }}^{\mathrm{H} / \mathrm{W}}$ involves the plaquettedriven Polyakov loop sector $\mathcal{F}_{\mathrm{P}}^{\mathrm{H} / \mathrm{W}}$ and the quark sector $\mathcal{F}_{\mathrm{Q}}^{\mathrm{H} / \mathrm{W}}$,

$$
\begin{aligned}
\mathcal{F}_{\text {eff }}^{\mathrm{H} / \mathrm{W}}\left(\Phi, \ell, \bar{\ell} ; \beta, m_{0}, T, \mu\right) \\
=\mathcal{F}_{\mathrm{P}}^{\mathrm{H} / \mathrm{W}}(\ell, \bar{\ell}, \beta, T)+\mathcal{F}_{\mathrm{Q}}^{\mathrm{H} / \mathrm{W}}\left(\Phi, \beta, m_{0}, T, \mu\right) \\
\quad+\mathcal{O}\left(1 / g^{6}, 1 / g^{2\left(N_{t}+2\right)}, 1 / \sqrt{d}\right) .
\end{aligned}
$$

The $\mathcal{F}_{\mathrm{P}}^{\mathrm{H} / \mathrm{W}}$ is responsible for the Polyakov loop effects

$$
L_{p}=N_{c}^{-1} \prod_{\tau} U_{0, \tau \mathbf{x}}, \quad U_{0}=\text { temporal link variable, }
$$

which result from the integral over the spatial link variables for the plaquettes wrapping around the temporal direction. Such Polyakov loops are dubbed "plaquette-driven," and purely gluonic. The effects of $L_{p}$ is investigated in two MFA scheme: the Haar measure and Weiss MFA-as indicated by the suffixes " $\mathrm{H}$ " and "W". In the former, the Polyakov loop $L_{p}$ is simply replaced with its constant mean-field $\ell$, while in the latter, the mean-field $\ell$ is introduced via the extended Hubbard-Stratonovich transformation [25] and the fluctuations from the mean-field is taken account in the integral over the $U_{0}$. The Polyakov loop effective potential of Haar measure MFA is wellknown since the 1980s [40],

$$
\begin{gathered}
\mathcal{F}_{\mathrm{P}}^{\mathrm{H}}(\ell, \bar{\ell}, \beta, T)=-2 T d N_{c}^{2}\left(\frac{1}{g^{2} N_{c}}\right)^{1 / T} \bar{\ell} \ell-T \log \mathcal{R}_{\mathrm{Haar}}, \\
\mathcal{R}_{\text {Haar }} \equiv 1-6 \bar{\ell} \ell-3(\bar{\ell} \ell)^{2}+4\left(\ell^{N_{c}}+\bar{\ell}^{N_{c}}\right),
\end{gathered}
$$

where the Haar measure in the $U_{0}$ path integral leads to the $Z_{3}$ symmetric term $\mathcal{R}_{\text {Haar }}$. Since the $\mathcal{R}_{\text {Haar }}$ does not couple to the dynamical quarks, the $Z_{3}$ symmetry affects the phase diagram separately from the chiral dynamics in Haar measure MFA. In sharp contrast to this, there is no counterpart in Weiss MFA [28],

$$
\mathcal{F}_{\mathrm{P}}^{\mathrm{W}}(\ell, \bar{\ell}, \beta, T)=2 T d N_{c}^{2}\left(\frac{1}{g^{2} N_{c}}\right)^{1 / T} \bar{\ell} \ell .
$$

The Polyakov loop effects other than the quadratic term (5) are entangled to the dynamical quarks in the quark determinant as explained in the followings. Thus, the $Z_{3}$ dynamics is totally spoiled by the dynamical quarks in Weiss MFA.

In both Haar measure and Weiss MFA cases, the order counting of the strong-coupling expansion reads,

$$
\mathcal{F}_{\mathrm{P}}^{\mathrm{H} / \mathrm{W}} \sim \mathcal{O}\left(\left(1 / g^{2}\right)^{N_{t}=1 / T}\right),
$$

and thus depends on the lattice temperature $T=1 / N_{t}$, which is subject to the integer value $N_{t}$. However in this paper, we regard $T$ as a continuous valued given number, which naturally follows in the lattice Matsubara formalism [41]. Around the chiral transition/crossover temperature $T_{c}$, we will show that the $\mathcal{F}_{\mathrm{P}}^{\mathrm{H} / \mathrm{W}}$ becomes comparable to the NLO or NNLO effects: $\mathcal{O}\left(1 / g^{2 / T_{c}}\right) \sim \mathcal{O}\left(1 / g^{2-4}\right)$.

The quark sector $\mathcal{F}_{\mathrm{Q}}^{\mathrm{H} / \mathrm{W}}$ in Eq. (1) is derived by integrating out the staggered quarks with link/plaquette variables in each order of the strong-coupling expansion. In this paper, we consider the LO, NLO, and NNLO effects;

$$
\mathcal{F}_{\mathrm{Q}}^{\mathrm{H} / \mathrm{W}} \ni \mathcal{O}\left(1 / g^{0}\right), \mathcal{O}\left(1 / g^{2}\right), \mathcal{O}\left(1 / g^{4}\right) .
$$

The integral is evaluated by introducing several auxiliary fields $\Phi$, which includes the chiral condensate $\sigma$, the order parameter of the $U_{\chi}(1)$ chiral symmetry, as well as other fields,

$\Phi=\left\{\sigma, \psi_{\tau}, \bar{\psi}_{\tau}, \psi_{s}, \bar{\psi}_{s}, \psi_{\tau s}, \bar{\psi}_{\tau s}, \psi_{s s}, \bar{\psi}_{s s}, \psi_{\tau \tau}, \bar{\psi}_{\tau \tau}\right\}$,

whose physical meanings are summarized in Tables I and II in the Appendix. The coefficients of the effective potential terms are solely characterized by $\left(\beta, N_{c}, d\right)$ and $\mathcal{O}\left(1 / g^{0-4}\right)$ (see Table III). The total quark sector $\mathcal{F}_{\mathrm{Q}}^{\mathrm{H} / \mathrm{W}}$ is then divided into the auxiliary field part $\mathcal{F}_{\mathrm{X}}$ and the quark determinant 
part $\mathcal{F}_{\text {det }}^{\mathrm{H} / \mathrm{W}}$. As shown in Eq. (A17) in the Appendix, the $\mathcal{F}_{\mathrm{X}}$ is composed of the quadratic terms of the auxiliary fields $\Phi$.

The quark determinant term $\mathcal{F}_{\text {det }}^{\mathrm{H} / \mathrm{W}}$ is responsible for the dynamical quark effects, and includes the quark hoppings with link variables $U_{0}$ wrapping around the temporal direction, which give rise to the "quark-driven" Polyakov loops. In Haar measure MFA, the quark determinant part becomes similar to that in the Polyakov-loop-extended Nambu-Jona-Lasinio (PNJL) model [42-44] and the Polyakov-loop-extended Quark-Meson (PQM) model [45]:

$$
\begin{aligned}
\mathcal{F}_{\mathrm{det}}^{\mathrm{H}}= & -N_{c} E_{q}-N_{c} \log \sqrt{Z_{+} Z_{-}} \\
& -T\left(\log \mathcal{R}_{q}\left(E_{q}-\tilde{\mu}, \ell, \bar{\ell}\right)+\log \mathcal{R}_{q}\left(E_{q}+\tilde{\mu}, \bar{\ell}, \ell\right)\right),
\end{aligned}
$$

$\mathcal{R}_{q}(x, y, \bar{y}) \equiv 1+N_{c}\left(y e^{-x / T}+\bar{y} e^{-2 x / T}\right)+e^{-3 x / T}$.

See Table IV for the quark excitation energy $E_{q}$, the shifted quark chemical potential $\tilde{\mu}$, and the wave function renormalization factor $\sqrt{Z_{+} Z_{-}}$. In Weiss MFA, the plaquette-driven and quark-driven Polyakov loops are combined in the quark determinant, and the $U_{0}$ path integral accounts for the Polyakov loop fluctuations. Then we obtain the following expression,

$$
\begin{gathered}
\mathcal{F}_{\mathrm{det}}^{\mathrm{W}}=-N_{c} \log \sqrt{Z_{+} Z_{-}}-T \log \left[\sum_{I} \mathcal{Q}^{I}(\Phi) \mathcal{P}^{I}(\ell, \bar{\ell})\right], \\
\mathcal{P}^{I}(\ell, \bar{\ell})=\sum_{n=-\infty}^{\infty}(\sqrt{\ell / \bar{\ell}})^{-N_{c} n+N_{\mathrm{Q}}^{I}} \mathcal{P}_{n}^{I}(\sqrt{\ell \bar{\ell}}),
\end{gathered}
$$

where the thermal excitation of a quark and its composite $\mathcal{Q}^{I}$, the thermal excitation of Polyakov loops $\mathcal{P}_{n}^{I}$, and the quark number index $N_{\mathrm{Q}}^{I}$ are summarized in Table $\mathrm{V}$ in the Appendix. In the heavy quark limit $m_{0} \rightarrow \infty$, Eq. (11) recovers the $Z_{3}$ symmetry as shown in the Appendix.

The auxiliary fields $\{\Phi, \ell, \bar{\ell}\}$ at equilibrium are determined as a function of $\left(\beta, m_{0}, T, \mu\right)$ via the saddle point search of the effective potential $\mathcal{F}_{\text {eff }}^{\mathrm{H} / \mathrm{W}}$. In particular, the important quantities to probe the phase diagram are the chiral condensate $\sigma \in \Phi$, Polyakov loops $(\ell, \bar{\ell})$, and their (dimensionless) susceptibilities $\left(\chi_{\sigma}, \chi_{\ell}\right)$. In the present mean-field framework, the susceptibilities are evaluated as follows: We consider the curvature matrix $C$ of the effective potential at equilibrium,

$$
C_{i j}=\left.\frac{1}{T^{4}} \frac{\partial^{2} \mathcal{F}_{\mathrm{eff}}^{\mathrm{H} / \mathrm{W}}}{\partial \phi_{i} \partial \phi_{j}}\right|_{\text {equilibrium }},
$$

where the field $\phi_{i}$ represents the dimensionless auxiliary fields normalized by $T$ and $N_{c}$,

$$
\begin{aligned}
\phi_{i} \in & \left\{\frac{\sigma}{T^{3} N_{c}}, \frac{\psi_{\tau}}{T^{3} N_{c}}, \frac{\bar{\psi}_{\tau}}{T^{3} N_{c}}, \frac{\psi_{s}}{T^{6} N_{c}^{2}}, \frac{\bar{\psi}_{s}}{T^{6} N_{c}^{2}},\right. \\
& \left.\frac{\psi_{\tau s}}{T^{6} N_{c}^{2}}, \frac{\bar{\psi}_{\tau s}}{T^{6} N_{c}^{2}}, \frac{\psi_{s s}}{T^{12} N_{c}^{4}}, \frac{\bar{\psi}_{s s}}{T^{6} N_{c}^{2}}, \frac{\psi_{\tau \tau}}{T^{3} N_{c}}, \frac{\bar{\psi}_{\tau \tau}}{T^{3} N_{c}}, \ell, \bar{\ell}\right\} .
\end{aligned}
$$

Then the chiral and Polyakov loop susceptibilities are given by

$$
\chi_{\sigma}=\left(C^{-1}\right)_{i j=\sigma \sigma}, \quad \chi_{\ell}=\left(C^{-1}\right)_{i j=\ell \bar{\ell}}
$$

In addition, we investigate thermodynamic quantities, a pressure $p$, quark number density $\rho_{q}$, and interaction measure $\Delta$,

$$
\begin{aligned}
p & =-\left(\mathcal{F}_{\mathrm{eff}}^{\mathrm{H} / \mathrm{W}}(T, \mu)-\mathcal{F}_{\mathrm{eff}}^{\mathrm{H} / \mathrm{W}}(0,0)\right), \\
\rho_{q} & =\frac{\partial p}{\partial \mu} \\
\Delta & =\frac{\epsilon-3 p}{T^{4}}
\end{aligned}
$$

where $\epsilon=-p+T s+\mu \rho_{q}$ represents an internal energy with $s=\partial p / \partial T$ being an entropy.

\section{RESULTS}

We investigate the QCD phase diagram based on the effective potential explained in the previous section. We show the phase diagram and related quantities obtained in Haar measure MFA at next-to-leading order (NLO) in Subsec. III A, Weiss MFA at NLO in Subsec. III B for the fixed lattice coupling $\beta=4$ in the chiral limit $\left(m_{0}=0\right)$. We extend our study to include the finite bare quark mass $m_{0}>0$ in Subsec. III C with a particular focus on the chiral and Polyakov loop susceptibilities. Then, in Subsec. III D, we show the phase diagram evolution for various $\beta$. Finally, in Subsec. III E, we study the next-to-next-to-leading order (NNLO) effects in the phase diagram. The quark mass $m_{0}$, temperature $T$, quark chemical potential $\mu$, and other quantities are all given in lattice units, unless explicitly stated otherwise.

\section{A. Haar measure MFA at NLO}

We consider the NLO Haar measure MFA, where the NNLO $\mathcal{O}\left(1 / g^{4}\right)$ terms in the coupling coefficients shown in Table III and the Polyakov loop fluctuations are ignored. We concentrate on the chiral limit case $m_{0}=0$. We take the lattice coupling $\beta=4.0$ as a typical value, for which the chiral transition temperature at vanishing quark chemical potential $T_{c, \mu=0}$ [28] becomes close to the LQCD-MC result [37] (For details on the comparison, see Refs. [28,31]). The effects ignored or restricted here will be investigated in 
later subsections. The phase diagram in the Haar measure MFA is partly studied in our previous work [26], and we provide more complete analyses in the followings.

In the upper panel of Fig. 2, we show the chiral condensates $\left(\sigma / N_{c}\right)$ and Polyakov loops $(\ell, \bar{\ell})$ at finite quark chemical potential $\mu=0.4$ as a function of temperature $T$ for $\left(\beta, m_{0}\right)=(4.0,0.0)$. In the low $T$ region, the chiral broken $(\sigma \neq 0)$ and confined $(\ell \sim 0)$ phase appears. As $T$ increases, we observe the second-order chiral phase transition $(\sigma \rightarrow 0)$ at $T_{c} \simeq 0.44$ and the large increase of the Polyakov loops $(\ell \rightarrow \mathcal{O}(1))$. These results are similar to the zero chemical potential case shown in the previous study [28].

We find that the Polyakov loop is smaller than the antiPolyakov loop $(\ell<\bar{\ell})$ in the chiral broken phase. This is understood from a quark screening effect at high density: A finite $\mu$ leads to a net quark number density at equilibrium, where putting additional quarks into the system would give a larger energy cost than antiquarks. Therefore the free energy of the quark gets larger than that of the antiquark
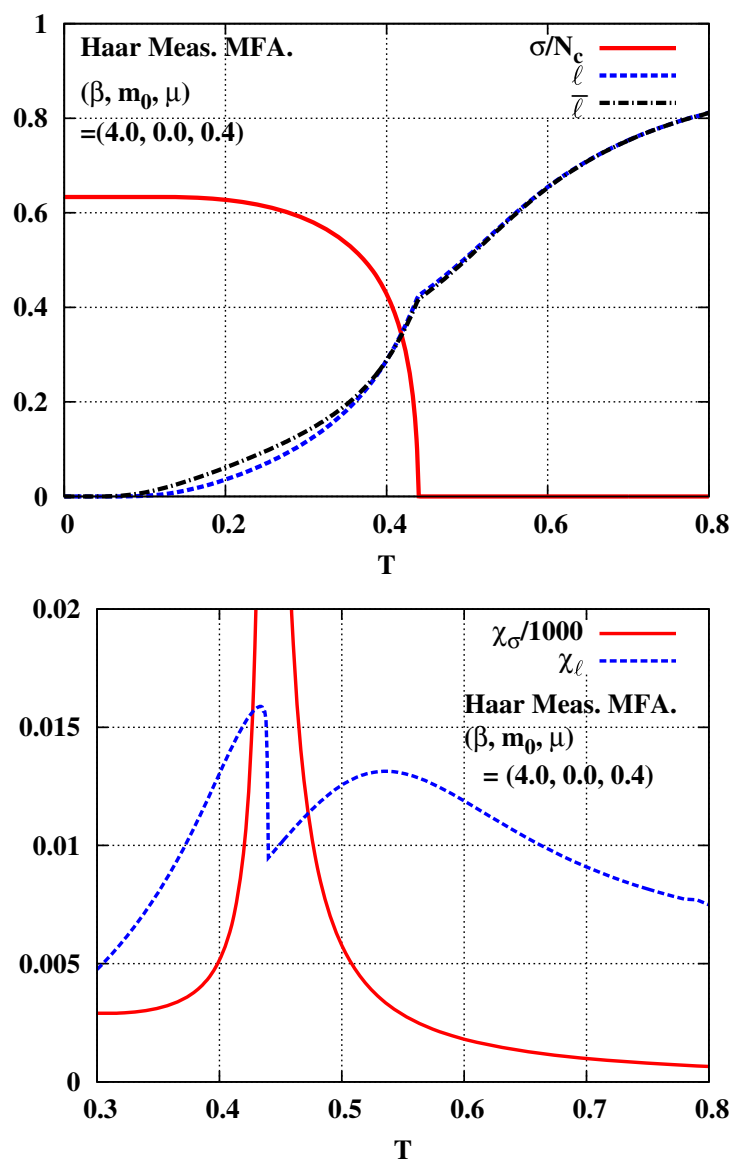

FIG. 2. Upper: The chiral condensates $\sigma$, Polyakov loops $(\ell, \bar{\ell})$, in NLO Haar measure MFA as a function of $T$ at $\left(\beta, m_{0}, \mu\right)=(4.0,0.0,0.4)$ in lattice units. Lower: The chiral and Polyakov loop susceptibilities $\left(\chi_{\sigma}\right.$ and $\left.\chi_{\ell}\right)$ in the same condition as the upper panel in lattice units. For a comparison, the $\chi_{\sigma}$ is multiplied by $1 / 1000$.
$F_{q}>F_{\bar{q}}$, which attributes to our observation $\ell<\bar{\ell}$ through the relation $(\ell, \bar{\ell}) \propto\left(e^{-F_{q} / T}, e^{-F_{\bar{q}} / T}\right)$.

In the lower panel of Fig. 2, we compare the temperature dependence of the chiral and Polyakov loop susceptibilities $\left(\chi_{\sigma}, \chi_{\ell}\right)$ which are defined in Eq. (15) in the same condition as the upper panel. The Polyakov loop susceptibility has two peaks with a relatively wide width. We note that the action in the present SC-LQCD (A2) has the $U_{\chi}(1)$ chiral symmetry, which governs the dynamics of the system. Since the first peak is found in the vicinity of the chiral phase transition, it should be associated with the chiral dynamics. For example, the $\chi_{\ell}$ rapidly (but continuously) decreases just after the peak, and its derivative with respect to $T$ is discontinuous. This property is associated by the second-order chiral phase transition,

$$
\sigma(T) \propto \begin{cases}\left(\frac{T_{c}-T}{T_{c}}\right)^{\beta_{\sigma}=1 / 2} & \left(T<T_{c}\right), \\ 0 & \left(T \geq T_{c}\right),\end{cases}
$$

via the potential curvature matrix Eq. (13). In general, fluctuation effects modifies the critical exponent $\beta_{\sigma}$, but the derivative is still discontinuous in the thermodynamic limit at the second-order transition. The second peak (or bump) is found in the chiral restored phase $T \simeq 0.53>T_{c}$, and interpreted as the remnant of the $Z_{3}$ deconfinement dynamics as discussed in the subsec. III C.

In the upper panel of Fig. 3, we show the chiral condensates $\sigma / N_{c}$ as a function of chemical potential $\mu$ for three fixed temperatures $T=0.15,0.20,0.25$. At $T=0.25$ (red-solid line), we find the second-order phase transition. At lower $T \sim 0.20$ (blue-dashed line), the chiral symmetry is partially restored with the first-order phase transition as $\mu$ increases, and gets completely restored with the second-order phase transition at larger $\mu$. As shown in the previous study [25], the partial chiral restoration (PCR) emerges due to the self-consistent evaluation of the finite $\beta$ effects for the chemical potential: The effective chemical potential appears as an implicit function of $\sigma, \mu \rightarrow$ $\tilde{\mu}(\sigma, \beta)=\mu-\delta \mu(\sigma, \beta)$ (see, Table IV), which allows a stable equilibrium satisfying $\sigma \sim \tilde{\mu}(\sigma)$, leading to the PCR. Our finding in the present study is that the PCR is not spoiled by the Polyakov loop effects, but still exists. As $T$ decreases, the PCR disappears and the first-order chiral transition dominates as indicated by the $T=0.15$ case (dashed-dotted black line).

In the lower panel of Fig. 3, we pick up the $T=0.25$ case from the upper panel and show the $\mu$ dependence of $\sigma / N_{c}$ in a wider range. The Polyakov loops $(\ell, \bar{\ell})$ and the quark number density $\left[\rho_{q} / N_{c}\right.$ defined by Eq. (17)] are also displayed. The Polyakov loops increase in the chiral broken phase $\mu<\mu_{c} \simeq 0.59$, and the increasing rate stays quite small compared with the finite $T$ transition case. In contrast, the quark number density rapidly increases in the vicinity of the chiral phase transition. After the 
MIURA, KAWAMOTO, NAKANO, and OHNISHI
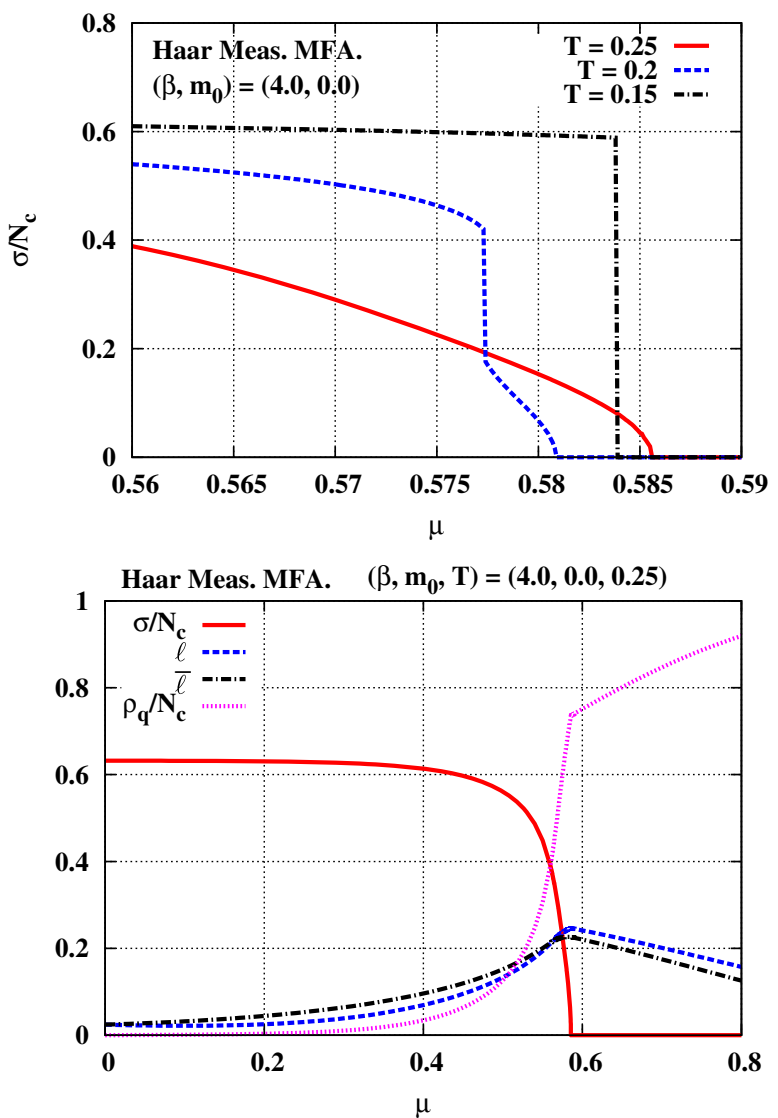

FIG. 3. Upper: The chiral condensates $\sigma$, Polyakov loops $(\ell, \bar{\ell})$, in NLO Haar measure MFA as a function of $\mu$ at $\left(\beta, m_{0}\right)=$ (4.0,0.0) for the selected temperatures $T=0.15,0.2,0.25$ in lattice units. Lower: The $\sigma,(\ell, \bar{\ell})$, and quark number density $\rho_{q}$ in NLO Haar measure MFA as a function of $\mu$ at $\left(\beta, m_{0}, T\right)=$ $(4.0,0.0,0.25)$ in lattice units.

transition $\left(\mu \geq \mu_{c}\right)$, we observe a high density system $\left(\rho_{q} \sim N_{c}\right)$ with a little quark excitation $(\ell \ll 1)$. This property as well as the possibility of two sequential transitions associated with the PCR would be reminiscent of the original idea of the quarkyonic phase [5].

In the symmetric phase, the Polyakov loops $(\ell, \bar{\ell})$ start decreasing with the relation $\bar{\ell}<\ell$ as $\mu$ increases. This would be a saturation artifact on the lattice: As we explained above, the chiral symmetry restoration leads to a high density system $\rho_{q}>N_{c} / 2$ so that more than half of the lattice sites are filled by quarks. Then the holes-sites without quarks - behave like antiquarks, and the system with the quark number density $\rho_{q}>N_{c} / 2$ would be identical to the system with the antiquark number density $\rho_{\bar{q}}=\left(N_{c}-\rho_{q}\right)<N_{c} / 2$. Therefore, the excitation property of quarks and antiquarks becomes opposite $\left(F_{q}<F_{\bar{q}}\right)$ as illustrated in Fig. 4, and thus $\bar{\ell}<\ell$ holds. As $\mu$ becomes larger after the half-filling, the number of holes decreases and the degrees of freedom get frozen. Hence the excitations of both quarks and antiquarks are suppressed at larger
PHYSICAL REVIEW D 95, 114505 (2017)

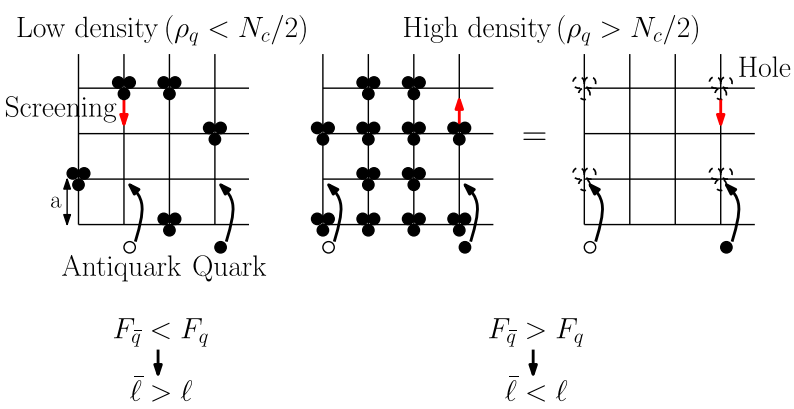

FIG. 4. The half-filled and saturation.

$\mu$, which results in the decreasing trend of $(\ell, \bar{\ell})$ as functions of $\mu$.

We show the phase diagram of NLO Haar measure MFA in the upper panel of Fig. 5 with $\left(\beta, m_{0}\right)=(4.0,0.0)$. The first-order chiral phase boundary (red-solid line) emerges in the low $T$ region and ends up with the tricritical point (TCP, filled black circle) at $\left(\mu_{\mathrm{TCP}}, T_{\mathrm{TCP}}\right) \simeq(0.577,0.205)$, from which the second-order chiral phase boundary (blue-dashed line) sets in with increasing temperature (The PCR emerges just below the TCP, and invisible in the resolution of Fig. 5 . The PCR becomes visible at larger $\beta$ as seen in Fig. 12). The lower-green (upper-yellow) band corresponds to the width of the Polyakov loop susceptibility $\chi_{\ell}$ at $90 \%$ of its
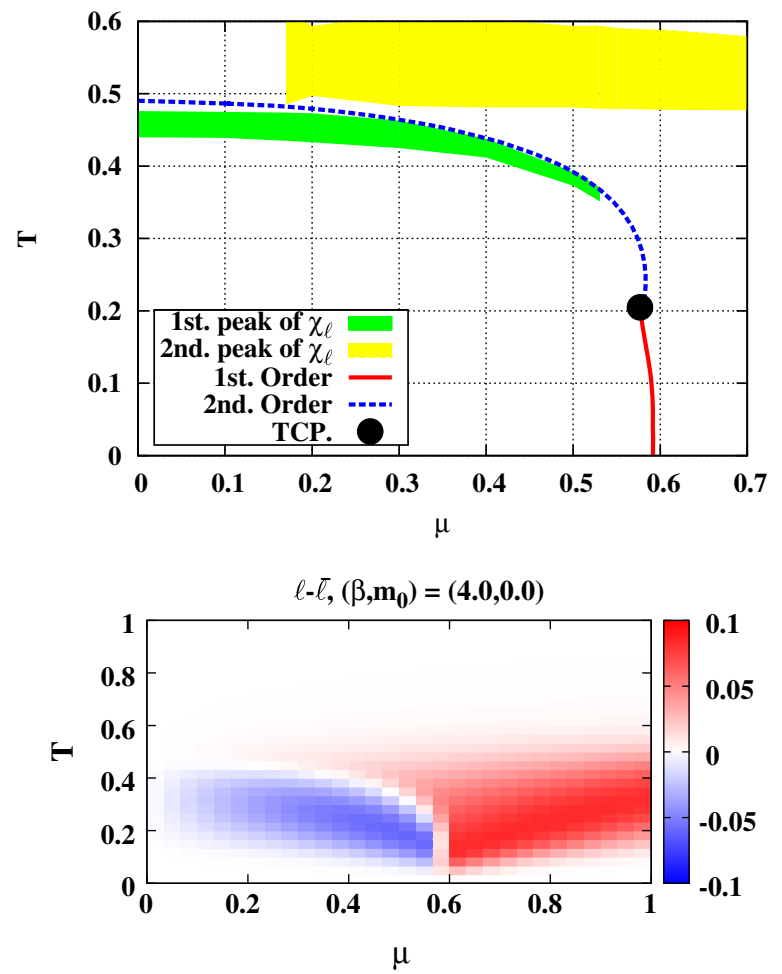

FIG. 5. Upper: The phase diagram of NLO Haar measure MFA at $\left(\beta, m_{0}\right)=(4.0,0.0)$ in lattice units. See texts for details. Lower: The difference of the Polyakov loop and anti-Polyakov loop in the phase diagram of the NLO Haar measure MFA. 
first (second) peak height. The first peak band depends on $\mu$ similarly to the chiral phase boundary: As mentioned above, the peak seems to be associated with the chiral phase transition. The peak strength becomes weaker with increasing $\mu$, and disappears at $\mu \simeq 0.53$ before reaching TCP. The second peak is almost independent of $\mu$, and starts appearing in $\mu \gtrsim 0.17$ separately from the first peak.

The phase diagram in the Haar measure MFA is similar to that in PQM [45]: When the $\mu$ dependence is absent in the Polyakov loop potential in PQM, the derivative of the Polyakov mean-field in terms of $T$ at finite $\mu$ has double peaks, which is analogous to our result shown in the lower panel of Fig. 2 as well as in our previous study [26]. We will revisit this subject in Weiss MFA case in the next subsection.

The lower panel of Fig. 5 shows the difference of the Polyakov loop and anti-Polyakov loop $(\ell-\bar{\ell})$ in the $T-\mu$ plane. The relation $\ell<\bar{\ell}$ holds in the whole $T, \mu>0$ region in the chiral broken phase as shown by the blue color. The saturation effect $\ell>\bar{\ell}$ is observed as a general tendency at large $\mu$ region in the chiral restored phase as indicated by the red color.

As shown in Eq. (6), the plaquette-driven Polyakov loop action includes the $\mathcal{O}\left(1 / g^{2 / T}\right)$ correction. At the chiral phase boundary, this effect gives $\mathcal{O}\left(1 / g^{2 / T_{c}}\right) \lesssim \mathcal{O}\left(1 / g^{4}\right)$. For the consistency of the strong coupling expansion, we have to take account of the NNLO $1 / g^{4}$ effects for the quark sector, which will be discussed in the later subsection.

\section{B. Weiss MFA at NLO}

We investigate the phase diagram of NLO Weiss MFA, where the Polyakov loop fluctuations from the mean fields $(\ell, \bar{\ell})$ are considered, while the NNLO effects $\mathcal{O}\left(1 / g^{4}\right)$ in the coupling coefficients shown in Table III are ignored. We compare the Weiss MFA results with the Haar measure MFA to clarify the effects of the Polyakov loop fluctuations to the phase diagram. We choose the same parameter set as the Haar measure MFA case, $\left(\beta, m_{0}\right)=(4.0,0.0)$.

As shown in Fig. $6, T$ or $\mu$ dependence of $\left(\sigma, \ell, \bar{\ell}, \rho_{q}\right)$ is qualitatively the same as the Haar measure MFA results. In the following, we concentrate on the results which are characteristic of the Weiss MFA.

In Fig. 7, we show the chiral and Polyakov loop susceptibilities $\left(\chi_{\sigma}, \chi_{\ell}\right)$ at finite chemical potential $\mu=$ 0.4 as a function of temperature $T$. Two peaks are almost degenerated, and the width of $\chi_{\ell}$ is sharper than the Haar measure MFA case. We do not see the second $\left(Z_{3}\right.$ associated) peak in the chiral symmetric phase in sharp contrast to the Haar measure MFA case.

In Fig. 8, we show the phase diagram of NLO Weiss MFA with $\left(\beta, m_{0}\right)=(4.0,0.0)$. We find two qualitative differences between the NLO Weiss MFA and NLO Haar measure MFA results: First, the peak of $\chi_{\ell}$ (green-band showing the width of $\chi_{\ell}$ at $90 \%$ of the peak height) is more strongly locked to the chiral phase boundary in Weiss MFA
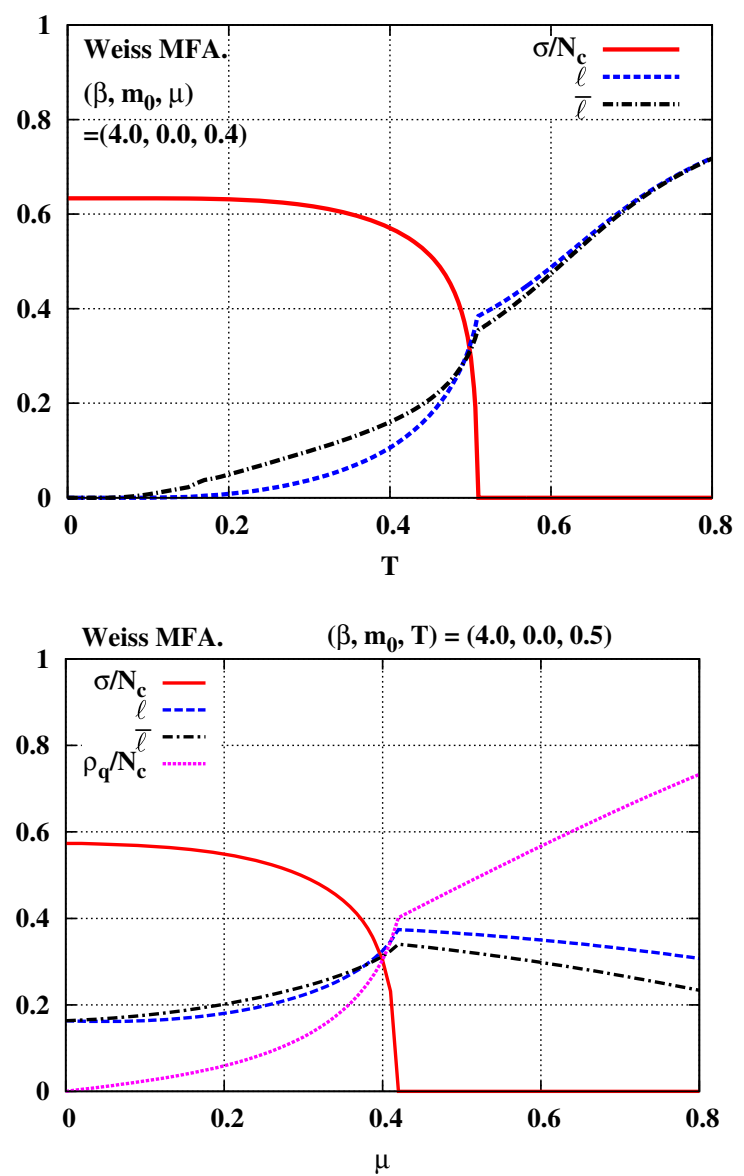

FIG. 6. Upper: The chiral condensates $\sigma$, Polyakov loops $(\ell, \bar{\ell})$, in NLO Weiss MFA as a function of $T$ at $\left(\beta, m_{0}, \mu\right)=$ $(4.0,0.0,0.4)$ in lattice units. Lower: The chiral condensates $\sigma$, Polyakov loops $(\ell, \bar{\ell})$, and quark number density $\rho_{q} / N_{c}$ in NLO Weiss MFA as a function of $\mu$ at $\left(\beta, m_{0}, T\right)=(4.0,0.0,0.5)$ in lattice units.

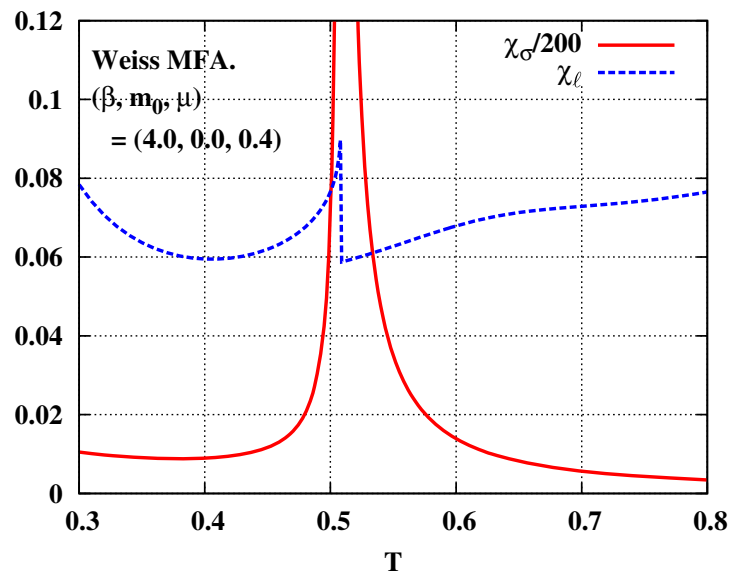

FIG. 7. The chiral and Polyakov loop susceptibilities $\left(\chi_{\sigma}, \chi_{\ell}\right)$ in NLO Weiss MFA as a function of $T$ at $\left(\beta, m_{0}, \mu\right)=(4.0,0.0,0.4)$ in lattice units. For a comparison, the chiral susceptibility $\chi_{\sigma}$ has been multiplied by $1 / 200$. 
MIURA, KAWAMOTO, NAKANO, and OHNISHI

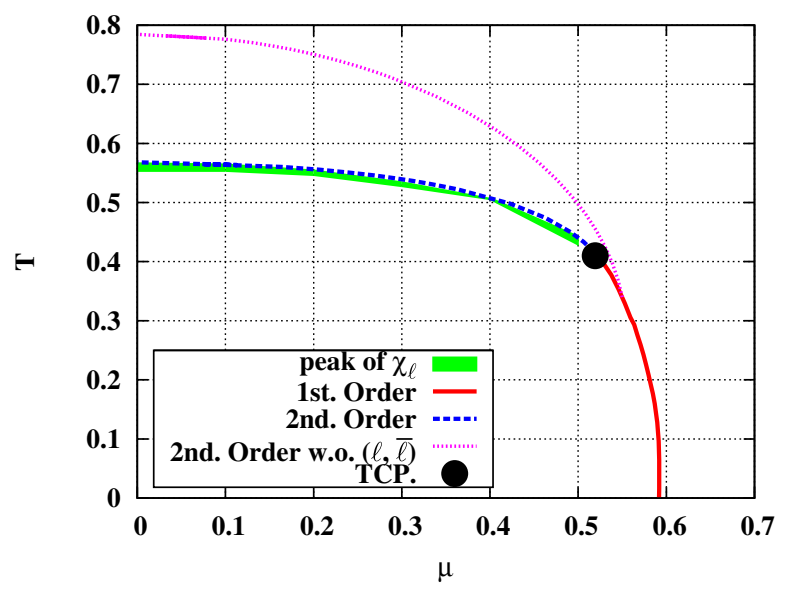

FIG. 8. The phase diagram at $\left(\beta, m_{0}\right)=(4.0,0.0)$ in NLO with Weiss MFA in lattice units. See texts for details.

than the Haar measure MFA case. Second, the remnant of the $Z_{3}$ dynamics such as the yellow band in Fig. 5 does not appear at any $\mu$ in the Weiss MFA case. As explained after Eq. (5) in the previous section, the plaquette-driven Polyakov loops are combined into the quark determinant and coupled to the dynamical quark effects via the $U_{0}$ path integral. Then, the Weiss MFA does not admit the remnant of the $Z_{3}$ symmetry in sharp contrast to the Haar measure MFA and many other chiral effective models $[42,43,45]$. It is sometimes argued that the chiral and deconfinement dynamics might be separated at finite $\mu$ [2], but the Weiss MFA does not support the isolated deconfinement dynamics from the chiral phase boundary.

Here, we comment on the recent phase diagram study by the PQM model [45]. In this model, a $\mu$ dependence was assumed in the Polyakov loop effective potential based on the phenomenological insights to describe the backreaction of the quark-matter to the Polyakov loops at finite density. This prescription led to a stronger locking between the peak of $d \ell / d T$ and the chiral crossover line, and the double peak structure of $d \ell / d T$ disappeared. These phenomena would be analogous to our findings in the Weiss MFA. We stress that the Weiss MFA effective potential directly results from the path integral in the lattice QCD without additional assumptions. This would be the advantage of the SCLQCD based effective potential.

We shall consider the formal limit $(\ell, \bar{\ell}) \rightarrow 0$ in the effective potential of Weiss MFA $\mathcal{F}_{\text {eff }}^{\mathrm{W}}$ : In the second line of Eq. (11), the thermal excitations (see Table V) carrying a quark number $0(I=\mathrm{MMM}, \mathrm{MQQ})$ and $\pm 3(I=\mathrm{B}, \overline{\mathrm{B}})$ remains and the $\mathcal{F}_{\text {eff }}^{\mathrm{W}}$ reduces into the effective potential which has been derived in our previous study [25]. We express the reduced effective potential as $\mathcal{F}_{\text {eff }}^{\mathrm{NLO}}$, and the results obtained by using $\mathcal{F}_{\text {eff }}^{\mathrm{NLO}}$ will be referred to as $N L O$ without Polyakov loops in the later discussions. See Eq. (A24) for the expression of $\mathcal{F}_{\text {eff }}^{\mathrm{NLO}}$. Needless to say, the $\mathcal{F}_{\text {eff }}^{\mathrm{NLO}}$ does not implement the Polyakov loop dynamics.
PHYSICAL REVIEW D 95, 114505 (2017)

By comparing the Weiss NLO MFA and the NLO without Polyakov loops, the Polyakov loop effects become more transparent.

In Fig. 8, we compare the chiral phase boundary of the NLO Weiss MFA and the NLO without Polyakov loops. The second-order phase boundary of the NLO Weiss MFA (bluedashed line) is found in lower $T$ region than that of NLO without Polyakov loop (magenta-dotted line). As $\mu$ becomes larger, two phase boundaries get closer to each other and degenerate in the vicinity of the TCP. The first-order phase boundary is almost independent of the Polyakov loop effects. This is understood as follows. As explained in the previous section, the plaquette-driven Polyakov loops gives the contribution of $\mathcal{O}\left(\left[1 / g^{2}\right]^{1 / T_{c}(\mu)}\right)$. At larger $\mu$, this factor decreases because the $T_{c}(\mu)$ does, and thus the Polyakov loop effects becomes higher order effects of the strongcoupling expansion, and thereby suppressed.

Compared with the Haar measure MFA, the transition temperature $T_{c}(\mu)$ in the Weiss MFA becomes somewhat larger. Then, the effect of the plaquette-driven Polyakov loops for $\beta=4.0$ is maximally $\mathcal{O}\left(\left[1 / g^{2}\right]^{1 / T_{c}(\mu=0)}\right)=$ $\mathcal{O}\left(1 / g^{3.3}\right)$, which is larger than the NNLO effects $1 / g^{4}$. Thus, the present NLO approximation for the quark sector is consistent with respect to the order counting of the strong coupling expansion, at least for $\beta \lesssim 4.0$.

Next, we investigate the thermodynamic quantities in the Weiss MFA. In the upper panel of Fig. 9, we show the normalized pressure $p / T^{4}$ as a function of $T$ at chemical potential $\mu=0.4$, the same condition as Fig. 7. In NLO Weiss MFA, the $p / T^{4}$ (red-solid line) becomes significantly larger at $T \gtrsim T_{c} \simeq 0.507$ and closer to the StefanBoltzmann result

$\frac{p_{\mathrm{sb}}}{T^{4}}=\frac{N_{f} N_{c}}{6}\left[\frac{7 \pi^{2}}{30}+\frac{\mu^{2}}{T^{2}}+\frac{1}{2 \pi^{2}} \frac{\mu^{4}}{T^{4}}\right]+\frac{\left(N_{c}^{2}-1\right) \pi^{2}}{45}$.

We do not see such a large enhance of $p / T^{4}$ in the case of NLO without Polyakov loops (blue-dashed line). Thus, the Polyakov loop plays an essential role to realize the pressure enhancement which is expected in the QGP phase at high $T$. More specifically, the pressure enhancement is attributed to the increase of Polyakov loop thermal excitations $\mathcal{P}_{n}^{I}(\sqrt{\ell \bar{\ell}})$ (see Table V) included in the Weiss MFA effective potential (11)-(12). This result should be compared with that in the PQM model, where the pressure is rather suppressed by Polyakov loops [45]. The different role of Polyakov loops is understood as follows. First, we recall that a usual NJL (QM) does not implement a confinement dynamics since quarks are introduced without gauge interactions. When Polyakov loop effects are introduced, giving PNJL (PQM), the Boltzmann factors for quark thermal excitations in the effective potential $[43,45]$ are multiplied by the Polyakov loop mean-field $\ell$, which acts as a suppression factor of the quark thermal excitations at low $T$. In this sense, the role of the Polyakov loop is to 

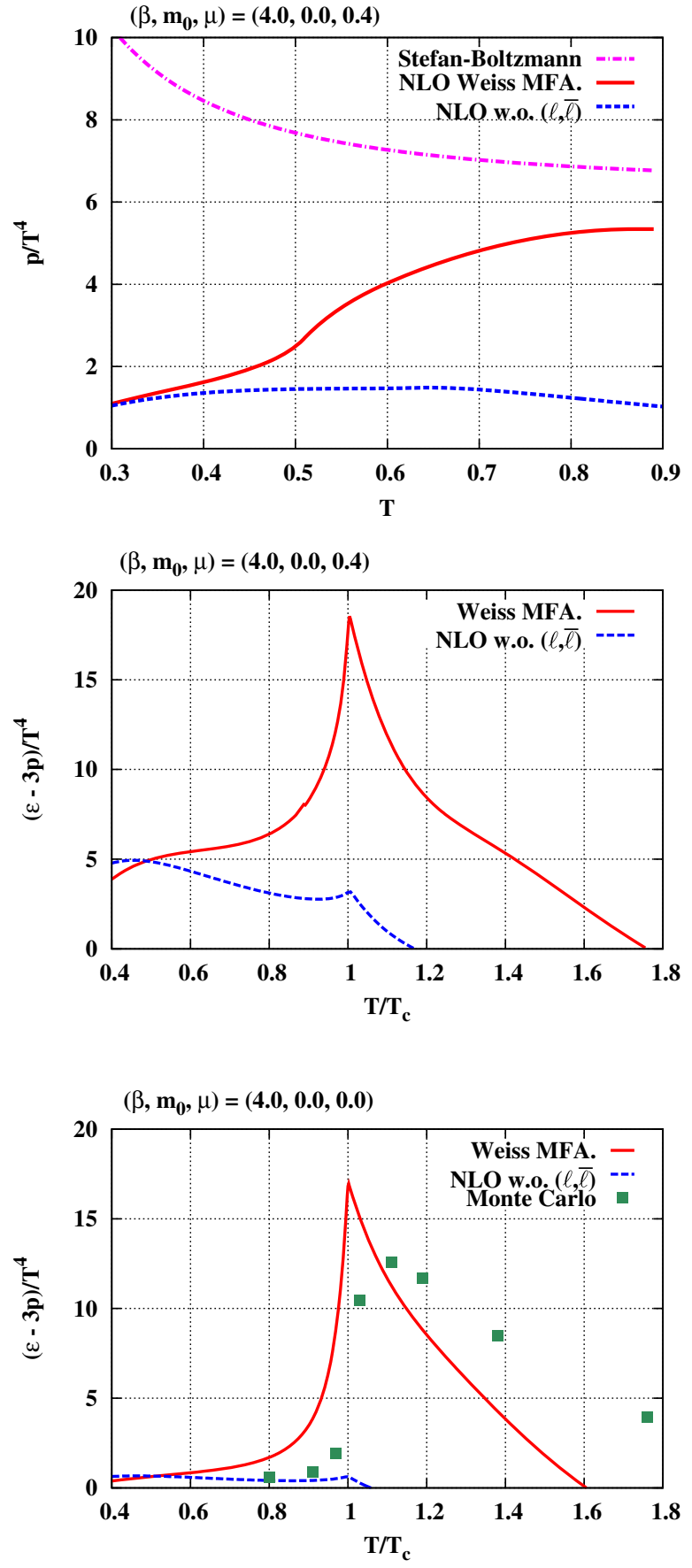

FIG. 9. The pressure (upper) and interaction measure (middle and lower) normalized by $T^{4}$ in "NLO Weiss MFA" and "NLO without Polyakov loops" as a function of $T$ at $\left(\beta, m_{0}, \mu\right)=$ $(4.0,0.0,0.4)$ (upper and middle), and $\left(\beta, m_{0}, \mu\right)=$ $(4.0,0.0,0.0)$ (lower). In the upper panel, the horizontal axis $T$ is in lattice units. In the middle and lower panels, the horizontal axis is normalized by critical temperature $T_{c}$. In the lower panel, we have quoted the Monte Carlo results [39].

confine quarks at low $T$ in PNJL and PQM, and therefore, suppresses the pressure. By comparison in SC-LQCD, the link integrals admit only color-singlet hadronic states contributing to the effective potential. As a result, the thermal excitations carrying the quark number $N_{\mathrm{Q}}^{I}= \pm 1$ (quark and antiquark excitations) and \pm 2 (diquark and antidiquark excitations) in Table V can emerge only when the Polyakov loop mean-fields are taken account. In this sense, the role of the Polyakov loop is to deconfine quarks at high $T$ in SC-LQCD, and enhances the pressure. Thus, Polyakov loops play different roles in the SC-LQCD and PNLO (PQM).

In the middle panel of Fig. 9, we show the interaction measure $\Delta=(\epsilon-3 p) / T^{4}$ as a function of $T$ at chemical potential $\mu=0.4$. In NLO Weiss MFA, the $\Delta$ has a large peak in the vicinity of the chiral phase transition $T \sim T_{c}$ as expected with regards to the increasing scale asymmetry in the strongly interacting quark-gluon plasma (sQGP). This should be compared with the result obtained in NLO without Polyakov loops (dashed-blue line) staying small and showing just a tiny bump structure at $T \sim T_{c}$. In the lower panel of Fig. 9, we compare our results on the interaction measure at vanishing of chemical potential with those obtained in the Monte Carlo simulations (four flavor, the chiral limit is taken) [39]. The Monte Carlo results (green boxes) show the drastic increase in the vicinity of the chiral phase transition. This feature is qualitatively reproduced by the NLO Weiss result (red-solid line), but not in the NLO without Polyakov loops (blue-dashed line). Around $T=T_{c}$, a singular behavior in the derivative of $(\epsilon-3 p) / T^{4}$ with respect to $T$ is seen only in the chiral limit. This behavior is associated with the second-order chiral phase transition as mentioned in Sec. III A.

\section{Quark mass dependence}

In the previous subsections, we have studied the phase diagram in the chiral limit $m_{0}=0$. In this subsection, we investigate the $m_{0}$ dependence of the chiral and Polyakov loop susceptibilities. We choose the same parameter set of $\beta=4.0$ and $\mu=0.4$ as previous subsections.

In the upper panel of Fig. 10, we show the chiral susceptibility $\chi_{\sigma}$ of the NLO Haar measure MFA as a function of $T$ for various bare quark mass $m_{0}$ at $(\beta, \mu)=(4.0,0.4)$. The peak position defines the chiral crossover temperature at finite $m_{0}$. The chiral dynamics becomes weaker as indicated by the attenuating peak with increasing $m_{0}$. In the lower panel of Fig. 10, we show the Polyakov loop susceptibility $\chi_{\ell}$ in the same condition as the upper panel. The double peak structure which we have shown in the chiral limit in Subsec. III A evolves into a single peak with increasing $m_{0}$. The single peak grows up in the heavy mass region $m_{0}=0.9$, and comes to be responsible for the $Z_{3}$ crossover. Consistently, the chiral susceptibility does not show any signal there as shown in the upper panel.

We notice that the $Z_{3}$ peak of $\chi_{\ell}$ at $m_{0}=0.9$ locates at the almost same temperature as the second peak appearing in the small mass region $m_{0} \lesssim 0.01$. This implies that the second peak originates from the remnant of the $Z_{3}$ 
MIURA, KAWAMOTO, NAKANO, and OHNISHI

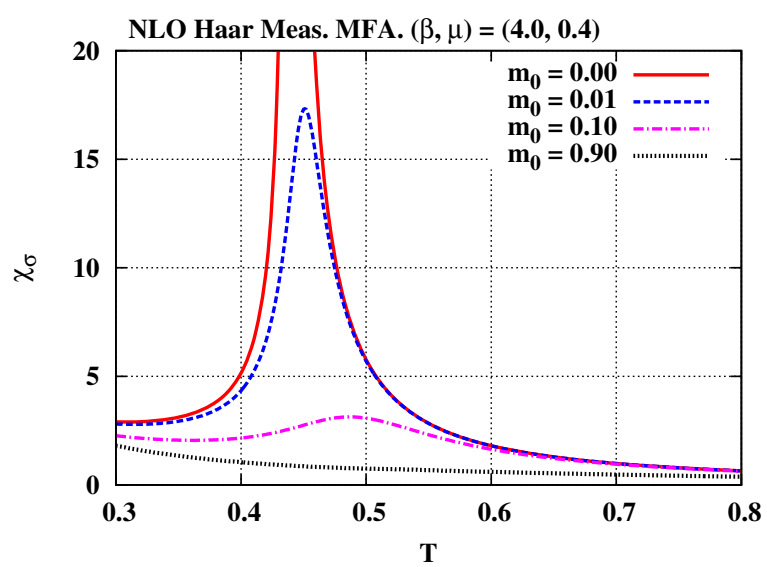

NLO Haar Meas. MFA. $(\beta, \mu)=(4.0,0.4)$

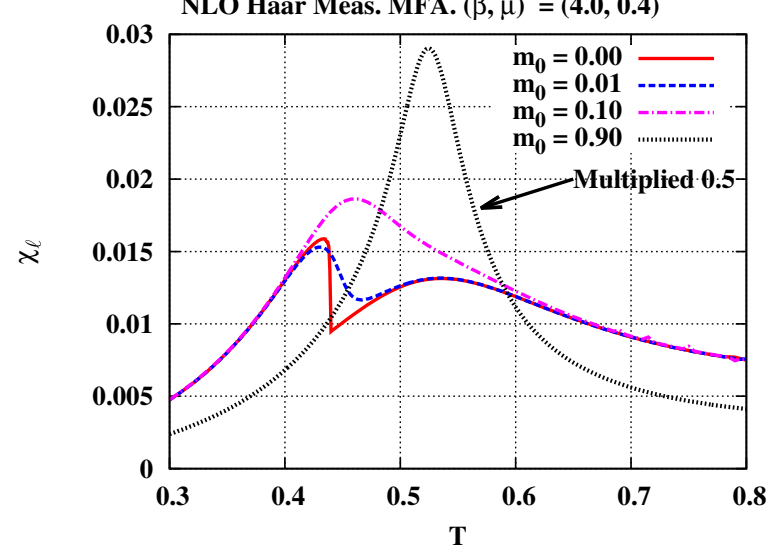

FIG. 10. The chiral (upper) and Polyakov loop (lower) susceptibilities as a function of $T$ for various bare quark mass $m_{0}$ at $(\beta, \mu)=(4.0,0.4)$ in NLO Haar measure MFA. All quantities are in lattice units.

dynamics. In fact, the approximate $Z_{3}$ symmetry remains even in the chiral limit in the effective potential of the Haar measure MFA: The $Z_{3}$ symmetric (Haar measure) term $\mathcal{R}_{\text {Haar }}$ in Eq. (4) has a large contribution and does not couple to the dynamical fermion effects $\mathcal{R}_{q}$ in Eq. (A18), so that the former effect is not horribly spoiled by the latter. The result is consistent with our previous work [26].

This should be compared with NLO Weiss MFA results, Fig. 11. The chiral susceptibility $\chi_{\sigma}$ (upper panel) is qualitatively the same as the Haar measure result, while the Polyakov loop susceptibility $\chi_{\ell}$ (lower panel) differs: The Weiss MFA does not lead to the double-peak structure in $\chi_{\ell}$ for any $m_{0}$. Thus, the scenario with the double-peak, or equivalently, the deconfinement separated from the chiral phase boundary at high density would be less supported within the present approximation. To extract a definite conclusion on the relation between two susceptibilities $\chi_{\sigma, \ell}$, we need to investigate the higher-order effects on the Polyakov loops.

It is worth mentioning that the Polyakov loop effective potential in the Haar measure MFA, Eq. (3) is similar to one of the popular choices of the potential in the PNJL model
PHYSICAL REVIEW D 95, 114505 (2017)

NLO Weiss MFA. $(\beta, \mu)=(4.0,0.4)$

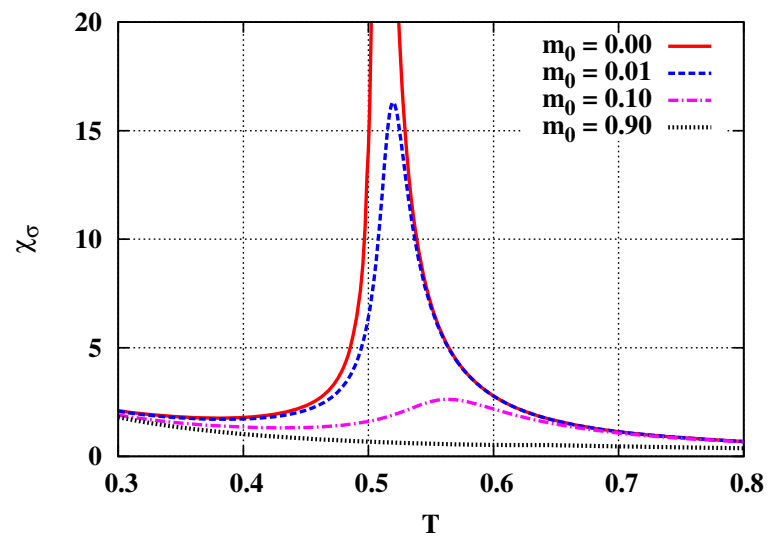

NLO Weiss MFA. $(\beta, \mu)=(4.0,0.4)$

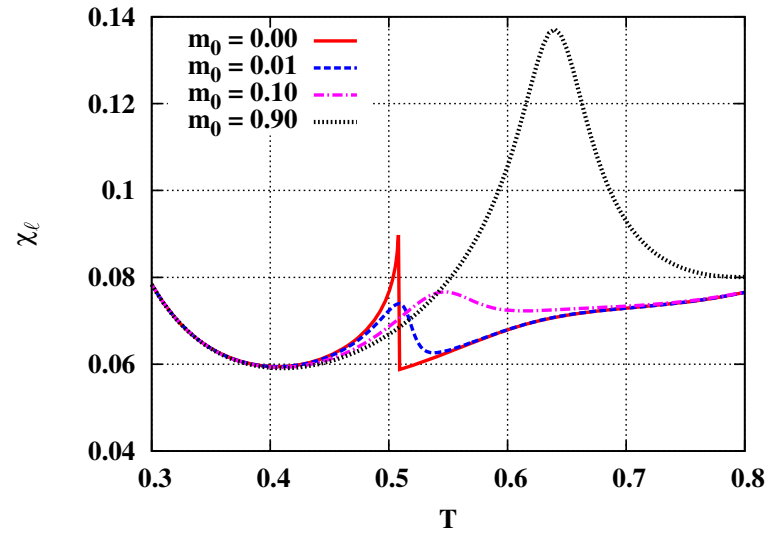

FIG. 11. The chiral (upper) and Polyakov loop (lower) susceptibilities as a function of $T$ for various bare quark mass $m_{0}$ at $(\beta, \mu)=(4.0,0.4)$ in NLO Weiss MFA. All quantities are in lattice units.

$[42,43]$ or PQM models. They could in principle contain the remnant of $Z_{3}$ dynamics as the Haar measure MFA does. As explained in the previous subsection, the recent work based on PQM assumed a certain $\mu$ dependence to the coefficients in the Polyakov loop effective potential [45]. This gives a phenomenological implementation of a back reaction from dynamical quark effects. The Weiss MFA effective potential [especially Eq. (11)] proposes the lattice QCD based solution for the quark back reaction to the Polyakov loops, and opens a possibility to upgrade the PNJL and PQM models so that they account for the Polyakov loop and quark degrees of freedom more systematically. To invent such a model based on the Weiss MFA should be one of the future works.

\section{Phase diagram evolution with increasing $\beta$}

So far, we have studied the phase diagram at a fixed coupling, $\beta=4.0$. In this subsection, we investigate the phase diagram for various lattice coupling ranging $0.0 \leq \beta \leq 6.0$, while we keep the vanishing bare quark mass $m_{0}=0$. For the chiral phase transition temperature 
at vanishing chemical potential $T_{c, \mu=0}$, the lattice $\mathrm{MC}$ data with one species of staggered fermion are available [30,37-39] and are compared with $T_{c, \mu=0}$ evaluated in the strong-coupling expansion $[25,27,28]$. We extend our analyses up to $\beta=6.0$, for which the physical scale of $\left(T_{c}, \mu_{c}\right)$ can be extracted by utilizing the lattice spacing result in Ref. [46].

In the upper panel of Fig. 12, we show the phase diagram evolution with increasing $\beta$ in the case of NLO Haar measure MFA. In the whole range of $0.0 \leq \beta \leq 6.0$, the chiral phase transition is a first-order in the low temperature region, and it evolves into the second-order at higher $T$ via TCP. The transition temperature at $\mu=0\left(T_{c, \mu=0}\right)$ acquires much larger modification with increasing $\beta$ than
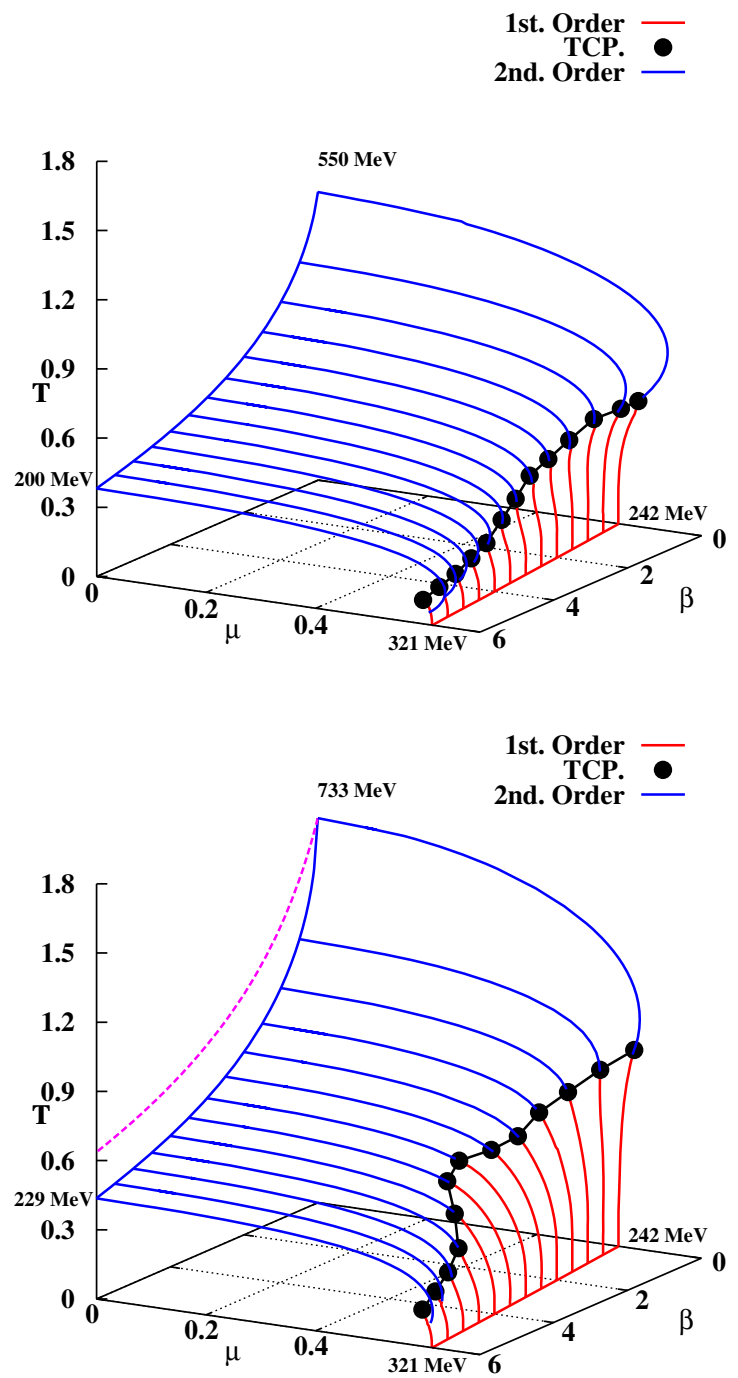

FIG. 12. The phase diagram evolution with the increasing lattice coupling $\beta$ at NLO Haar measure (upper) and Weiss (lower) MFA in the chiral limit. We have quoted $a^{-1}(\beta=0)=$ $440(\mathrm{MeV})$ and $a^{-1}(\beta=6)=524(\mathrm{MeV})$ from Ref. [46]. In Weiss MFA (lower), we have also shown our previous result on the transition temperature at $\mu=0$ without effects of plaquettedriven Polyakov loops [25] by the magenta-dashed line. the transition chemical potential at $T=0 \quad\left(\mu_{c, T=0}\right)$. Resultantly, the ratio $R=\mu_{c, \mathrm{~T}=0} / T_{c, \mu=0}$ which characterize the shape of the chiral phase boundary is greatly enhanced. For $\beta \geq 4$, the first-order transition line goes inside of the second-order transition line near the TCP, and the PCR explained in the previous subsection emerges between two lines.

In the lower panel of Fig. 12, we show the phase diagram evolution of the NLO Weiss MFA in the chiral limit $m_{0}=0.0$. The results are qualitatively same as the Haar Measure MFA case.

We compare the ratio $R=\mu_{c, \mathrm{~T}=0} / T_{c, \mu=0}$ of NLO Weiss MFA to that obtained in the "NLO without Polyakov loops". At $\beta=4.0(6.0)$, the former (red-solid line) in Fig. 13 becomes 1.38 (1.46) times larger than the latter (blue-dashed line). Thus the ratio $R$ becomes larger by the Polyakov loop effects. Next, we compare our $R$ with those obtained by the Monomer-Dimer-Polymer (MDP) simulation [31]. The MDP (green triangles in Fig. 13) gives a somewhat larger $R$ than our MFA result in the strongcoupling limit, and becomes closer to the NLO Weiss MFA at finite $\beta$. The increasing $R$ at larger $\beta$ is a common trend in both MFA and MDP, and preferable to be consistent with a realistic QCD phase diagram.

In both Haar measure MFA and Weiss MFAs, the TCP tends to go into low $T$ region with increasing $\beta$, and the second-order chiral phase boundary becomes dominant. However, the TCP and PCR evolution at large $\beta$ in Fig. 12 may be modified by a number of effects which are missing in the present mean-field framework at NLO; (1) fluctuation degrees of freedom from mean-fields, (2) effects of higherorder of the strong-coupling expansion, and (3) chiral anomaly effects. In the following, we discuss these corrections in relation to the continuum limit.

The fluctuation effects become important in critical phenomena and may give a non-negligible correction to

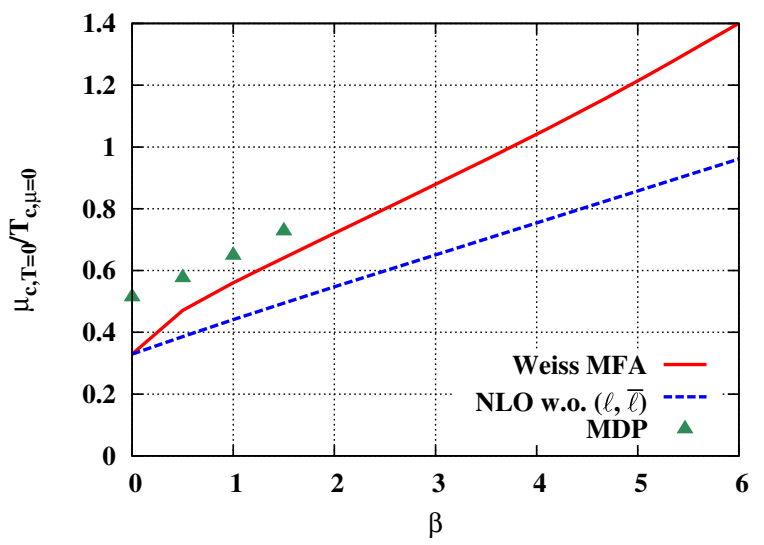

FIG. 13. The ratio $R=\mu_{c, \mathrm{~T}=0} / T_{c, \mu=0}$ as a function of the lattice bare coupling $\beta=2 N_{c} / g^{2}$. For a comparison, we show the MDP results for $R$ in Ref. [31]: We read off the $\mu_{c, T=0} \simeq 0.72$ from Fig. 6 in Ref. [31], and quoted the $T_{c}(\beta)$ evaluated by using the "exponential extrapolation" to calculate the $R$. 
the TCP and PCR obtained in MFA even at a fixed order of the strong-coupling expansion. This is the ambiguity (1). However, at least for the strong-coupling region $\beta \leq 1.5$, the basic property of the TCP shown in this work would be stable against the fluctuations; our results show that the TCP exists and it is almost independent of $\beta$, which is shown to remain intact even including the fluctuation effects $[32,33]$. For the evolution of TCP/PCR at larger $\beta$, only mean-field results (the present and previous works $[25,27])$ are available, and it is desirable to investigate the fluctuation effects in the near future.

If we assume that the (T)CP (and thereby, the first-order chiral phase transition) remains in the phase diagram in the continuum limit, the SC-LQCD including the fluctuation effects may have a contact to the critical phenomena expected in the continuum limit. This relies on the following reasoning. The SC-LQCD with one species of the staggered fermion has $O(2)$ symmetry at finite lattice coupling, while the massless two-flavor QCD in the continuum has $O(4)$. Since the sign of the relevant critical exponent in $O(2)$ is the same as that in $O(4)$, the ratio of various cumulants for the net baryon number $\left(\chi_{\mu}^{(n)} \propto \partial^{n} \log \mathcal{Z} / \partial\left(N_{c} \mu\right), \mathcal{Z}=\right.$ partition function) would be similar to each other. The cumulant ratio has been investigated only in the strong-coupling limit [47]. The finite coupling extension is, in principle, possible by combining the present study with the auxiliary-field Monte Carlo formulation [33].

Let us move on to the ambiguity (2), effects of higherorder of the strong-coupling expansion. We first comment on the remarkable properties at NLO; the first-order phase boundary diminishes with increasing $\beta$ as shown in Fig. 12, and this trend becomes rather significant at finite quark mass. For example in the Haar measure MFA, we found that the $C P$ associated angle $\arctan \left(T_{\mathrm{CP}} / \mu_{\mathrm{CP}}\right)$ at $\beta=4.0$ is 0.34 at $m_{0}=0.0$ and down to 0.31 at $m_{0}=0.05$. Thus, both of the increasing $\beta$ and nonzero $m_{0}$ disfavor the firstorder transition at NLO. The question is a fate of the above properties with higher-orders. To shed light on this, we quote the LQCD-MC results on the chiral critical surface [48] in the $\mu$-extended Columbia plot, where the surface evolution at finite $\mu$ implies the absence of the $C P$ at physical point mass. Thus, the properties at NLO explained above seems to be compatible to the LQCD-MC results including all order of $\beta$. This implies that the qualitative feature of the (T)CP at NLO would not be horribly changed by higher order effects. Of course, this naive expectation should be confirmed by investigating the higher-orders in future works. We note that the absence of $C P$ at physical point mass does not necessarily means the absence of critical phenomena, and the above discussion of the cumulant ratio for the ambiguity (1) can be compatible to the discussion here.

According to the effective model [49], the chiral phase transition in the $N_{f}=4>2$ system is predicted to be the first-order due to the chiral anomaly in the chiral limit. In the SC-LQCD with staggered fermions, however, the chiral anomaly is cancelled out among the species doublers and therefore missing in the present study. This is the ambiguity (3), and the anomaly effect may modify the properties of the TCP/PCR presented in this work. To shed light on this issue, we need to develop the SC-LQCD formulation with overlap fermions. We find some pioneering works [50]; it was argued that a massive flavor-singlet pseudoscaler meson could appear in SC-LQCD from a Jacobin term associated with a chirally-covariant transformation of the path-integral measure over quark fields. This was interpreted as a solution to the U(1) problem in the SC-LQCD context [50]. Thus, the Jacobian term seems to play an essential role to remedy the anomaly problem in SC-LQCD but has not been investigated in the literature of finite $T$ and/or $\mu$ (cf., [51]). This should also be the subject studied in future.

Finally, we estimate the $\left(T_{c}, \mu_{c}\right)$ in physical units by quoting the lattice spacing scale $a^{-1}(\beta=0)=440(\mathrm{MeV})$ and $a^{-1}(\beta=6)=524(\mathrm{MeV})$ from the zero temperature strong-coupling expansion [46]. In Haar measure MFA, we find $\left(T_{c, \mu=0}, \mu_{c, T=0}\right) \simeq(550,242)(\mathrm{MeV})$ in the strongcoupling limit, and $\left(T_{c, \mu=0}, \mu_{c, T=0}\right) \simeq(200,321)(\mathrm{MeV})$ at $\beta=6$. In Weiss MFA, we find $\left(T_{c, \mu=0}, \mu_{c, T=0}\right) \simeq$ $(733,242)(\mathrm{MeV})$ in the strong-coupling limit, and $\left(T_{c, \mu=0}, \mu_{c, T=0}\right) \simeq(229,321)(\mathrm{MeV})$ at $\beta=6.0$. Although the flavor-chiral structure of the present system differs from the real-life QCD, it is still interesting that the transition temperature of SC-LQCD gets closer to the realistic one $T_{c}^{\mathrm{MC}}=145-195 \mathrm{MeV}$ [3].

\section{E. Haar measure MFA at NNLO}

We investigate the phase diagram in the NNLO Haar measure MFA, where the $\mathcal{O}\left(1 / g^{4}\right)$ terms in the coupling coefficients (Table III) are considered. We adopt the same parameter set $\left(\beta, m_{0}\right)=(4.0,0.05)$ as that adopted in the previous work [28]. We investigate the property of the chiral condensates and the Polyakov loops at intermediate and high density region: $\mu=0.4$ and 0.7 . We compare the NNLO phase diagram with the NLO one, and studies the impact of the NNLO corrections.

In Fig. 14, we show the chiral condensate and Polyakov loop as a function of $T$ for the lattice coupling $\beta=4.0$. First, we consider the $\mu=0.4$ cases. At low $T$, the chiral symmetry is spontaneously broken $\left(\sigma / N_{c} \gg m_{0}=0.05\right.$, red-solid line), and the quarks are confined $(\ell \ll \mathcal{O}(1)$, blue-solid line). At high $T$, the chiral symmetry gets restored up to the finite bare mass effect $\left(\sigma / N_{c} \rightarrow \mathcal{O}\left(m_{0}\right) \sim 0.05\right)$, and the quarks becomes deconfined $(\ell \sim \mathcal{O}(1))$. The chiral condensate rapidly but smoothly decreases with increasing $T$, which indicates the chiral crossover rather than the phase transition. At larger chemical potential $\mu=0.7$, the chiral condensate (red-dashed line) is small and comparable to the 
NNLO Haar Measure MFA. $\left(\beta, \mathrm{m}_{0}\right)=(\mathbf{4 . 0}, 0.05)$

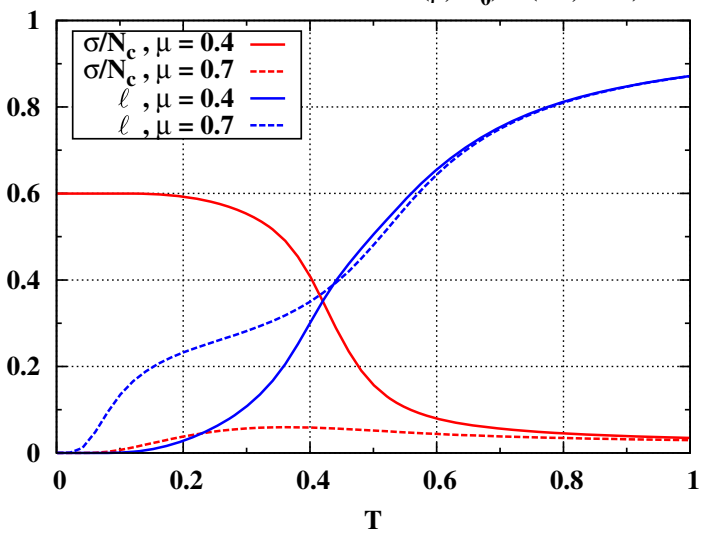

FIG. 14. Chiral condensates and Polyakov loops in the NNLO Haar measure MFA for $\beta=4.0$ with $m_{0}=0.05$ as a function of temperature at the two chemical potentials $\mu=0.4$ and 0.7 . All quantities are in lattice units.

bare quark mass $m_{0}=0.05$ in all $T$ region, and thus the chiral crossover is absent.

In Fig. 14, we find the clear difference in the Polyakov loop $\ell$ at $\mu=0.7$ and 0.4 ; the former (blue-dashed line) starts increasing even at a tiny (nonzero) temperature where the latter (blue-solid line) still remains small. This can be understood in terms of the presence/absence of the spontaneous breaking of the chiral symmetry; at $\mu=0.4$, the broken chiral symmetry leads to the dynamical quark mass and suppresses the thermal excitation of the quarks, while at 0.7 , there is no suppression due to the symmetry restoration. Thus, the relatively large $\ell$ at low temperature can be a characteristic feature at high density phase. At higher $T, \ell$ at $\mu=0.7$ becomes comparable with that at $\mu=0.4$.

In Fig. 15, we show the chiral susceptibility $\chi_{\sigma}$ at $\beta=4.0$ as a function of temperature $T$ for various chemical potential $\mu$. The peak position of the $\chi_{\sigma}$ locates a chiral

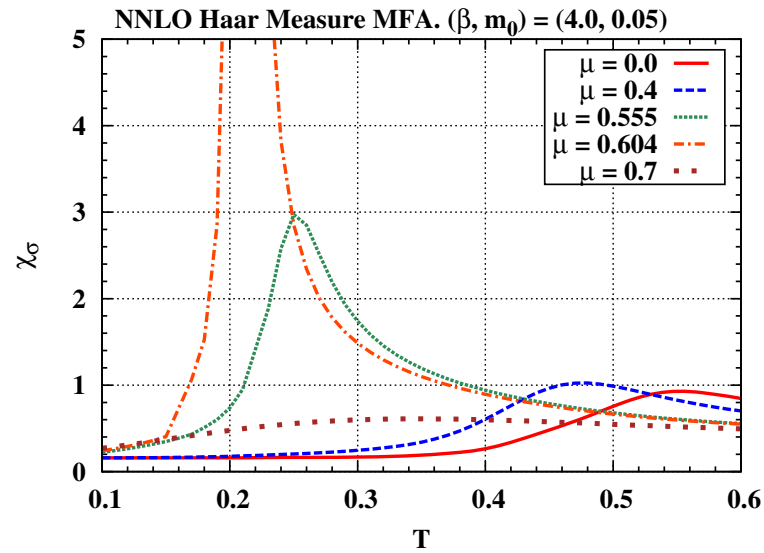

FIG. 15. The chiral susceptibility in NNLO Haar measure MFA with $m_{0}=0.05$ as a function of temperature for various $\mu$. All quantities are in lattice units at the coupling $\beta=4.0$. crossover and a critical endpoint (CEP). As $\mu$ increases from zero, the peak becomes gradually larger and moves to the smaller $T$ direction. When the $\mu$ reaches around 0.6 , the susceptibility shows a drastic enhancement, which indicates a critical phenomena associated with the CEP. At larger $\mu$, say 0.7 , the system is in the high-density phase where a peak is not seen for any $T$.

In Fig. 16, we show the temperature derivative of the chiral condensate and Polyakov loop as a function of temperature at $\mu=0.4$ and 0.7 . The lattice coupling $\beta$ is fixed at 4.0. At $\mu=0.4$ (solid lines), the chiral and deconfinement crossovers almost simultaneously take place as indicated by their peak positions. This property has been observed at $\mu=0$ [28]. Our finding here is that the locking of the chiral and deconfinement crossovers remains intact at finite $\mu$ as long as the spontaneous symmetry breaking exists. At $\mu=0.7, d\left(\sigma / N_{c}\right) / d T$ (red-dashed line) shows no signal at any $T$ due to the absence of the chiral crossover, and the $d \ell / d T$ (blue-dashed line) tends to lose peaklike structure.

In our previous studies [28], we have shown that the NNLO effects to the chiral phase transition/crossover at $\mu=0$ are very small. We shall now investigate the impact of the NNLO effects to the phase diagram including finite $\mu$. In the left panel of Fig. 17, we show the phase diagram evolution as a function of $\beta$ in NNLO Haar measure MFA. The black points represent the CEP which separates the chiral crossover region (higher $T$, blue-solid lines) and the first-order transition region (lower $T$, red-solid lines). Due to the finite coupling effects, the crossover line and the critical point move in the lower $T$ direction. For comparison, we show the counterpart at NLO with $m_{0}=0.05$ in the right panel. It is seen that the NNLO phase diagram (left) is very close to the NLO one (right).

In the end of the subsec. III A, we have mentioned that the NNLO effects for the quark sector should be included, particularly in the Haar measure MFA, to be consistent with

\section{NNLO Haar Measure MFA. $\left(\beta, \mathrm{m}_{0}\right)=(\mathbf{4 . 0}, 0.05)$}

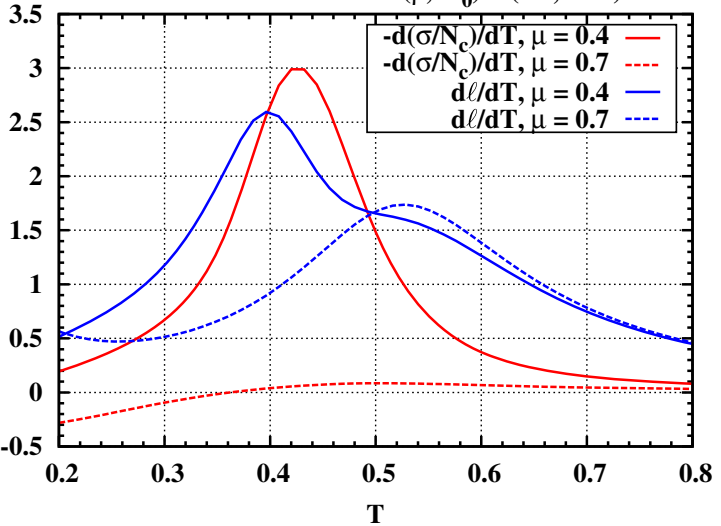

FIG. 16. The temperature derivative of the chiral condensates and Polyakov loops in the NNLO Haar measure MFA with $m_{0}=0.05$ as a function of temperature at $\mu=0.4$ and 0.7. All quantities are in lattice units at the coupling $\beta=4.0$. 


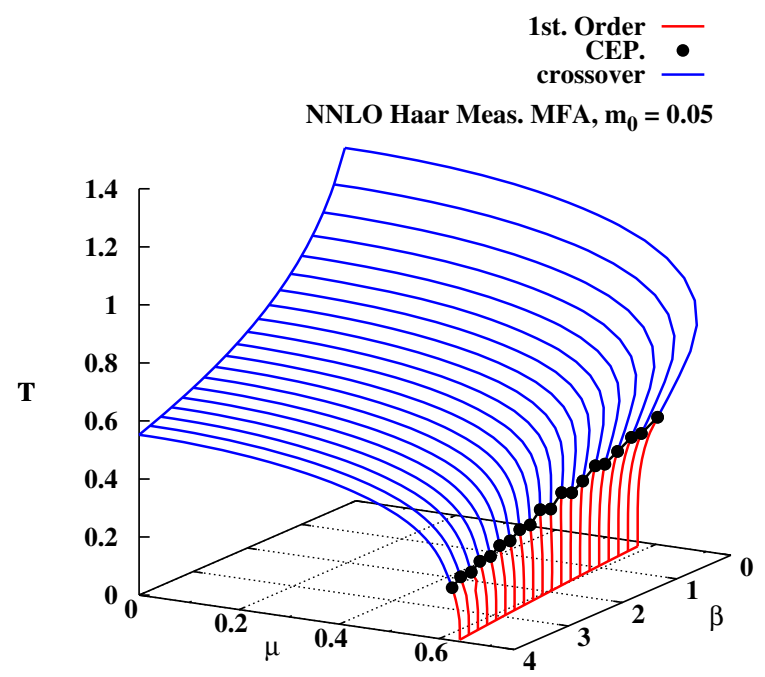

T

NLO Haar Meas. MFA, $\mathrm{m}_{0}=0.05$
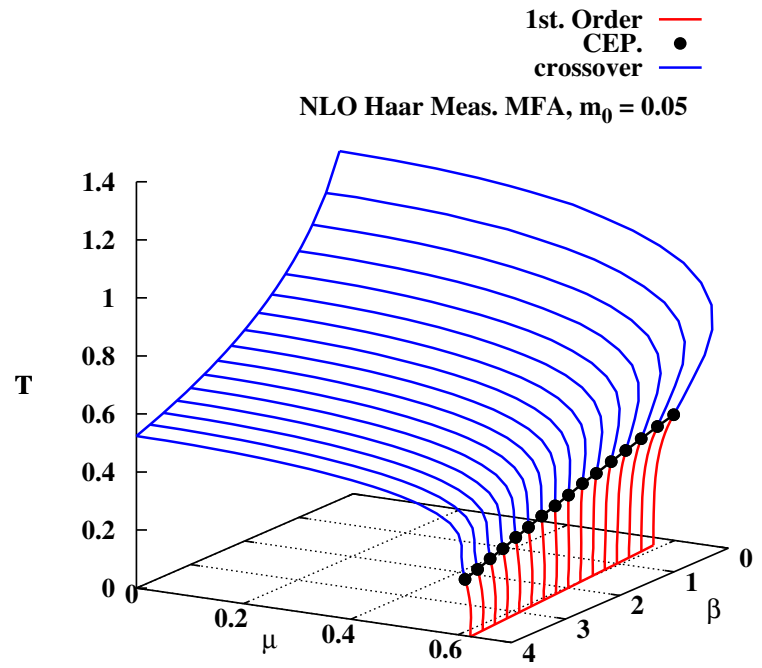

FIG. 17. The phase diagram evolution for $m_{0}=0.05$ as a function of $\beta$ in NNLO Haar-measure MFA (left), which is compared with the counterpart in NLO Haar-measure MFA (right) with same parameters. The red and blue lines represent the first-order chiral transition and the chiral crossover, respectively. The black points show the CEP separating the first-order and crossover phase boundaries. The little fluctuations of the CEP in the NNLO case are due to limited precision in the numerical search of the maximum of chiral susceptibilities as a function of $\mathrm{T}$ and mu. All quantities are in lattice units at a given $\beta$.

the plaquette-driven Polyakov loop sector with respect to the order counting of the strong-coupling expansion. However, the results in this subsection indicate that the NNLO corrections are tiny in whole region of the phase diagram. Thus, the NLO results shown in the previous subsections would be reliable.

\section{SUMMARY}

We have investigated the QCD phase diagram in color $\mathrm{SU}\left(N_{c}=3\right)$ gauge group at finite temperature $T$ and quark chemical potential $\mu$ by using the strong-coupling expansion of the lattice QCD (SC-LQCD) with one species of staggered fermion. Our effective potential [28] includes the LO $\left[\mathcal{O}\left(1 / g^{0}\right)\right]$, NLO $\left[\mathcal{O}\left(1 / g^{2}\right)\right]$, and NNLO $\left[\mathcal{O}\left(1 / g^{4}\right)\right]$ effects of the strong-coupling expansion in the quark sector, and the LO effects of Polyakov loop $\mathcal{O}\left(\left[1 / g^{2}\right]^{1 / T}\right)$ in the pure gluonic sector. The Polyakov loops are evaluated in two approximation schemes; a simple mean-field treatment [Haar measure mean-field approximation (MFA)] and an improved treatment with fluctuation effects (Weiss MFA). In this setup, we have investigated the whole structure of the SC-LQCD phase diagram with a special emphasis on the Polyakov loops effects.

In both Haar measure and Weiss MFA schemes, the firstorder chiral phase boundary emerges in the low $T$ region and ends up with the tricritical point (TCP), from which the second-order chiral phase boundary evolves to the smaller $\mu$ direction with increasing $T$ in the chiral limit $\left(m_{0}=0\right)$. The Polyakov loop together with finite $\beta$ effects strongly suppresses the critical temperature $T_{c}$ in the second-order/ crossover region at small $\mu$, while it gives a minor modification of the first-order phase boundary at larger $\mu$. As a result, the chiral phase boundary becomes much closer to the expected one in the real-life QCD as summarized in Fig. 12 (NLO case) and Fig. 17 (left: NNLO, right NLO). It is also remarkable that the NNLO effects are subdominant in whole region of the phase diagram.

In both Haar measure MFA and Weiss MFAs, the critical point $(C P)$ tends to go into low $T$ region with increasing $\beta$, and the second-order chiral phase boundary becomes dominant. This trend is also reported in the MDP simulations [31,32] and supports the recent MC results based on the critical surface analysis [48]. However, the trend is opposite to the anomaly based expectation for $N_{f}=4>2$ [49]. The anomaly effects in the staggered fermion formalism should be further investigated in the future.

We have investigated thermodynamic quantities, which is of great interest in the study of EOS of quark matter, which has however been challenging in SC-LQCD. Our findings are that a pressure and an interaction measure are drastically enhanced by Polyakov loop thermal excitations.

We have found some characteristic features of Polyakov loops at finite $\mu$. At finite $\mu$ in the broken phase, the antiPolyakov loop $\bar{\ell}$ becomes larger than $\ell$, which is interpreted as a screening effect of quarks at equilibrium with net quark number density. In the chirally symmetric high density phase, the Polyakov loop becomes relatively large even at a small temperature, which can be understood from the absence of the dynamical quark mass in the symmetric phase.

We have shown that the chiral and Polyakov loop susceptibilities $\left(\chi_{\sigma}, \chi_{\ell}\right)$ have their peaks near to each other in the second-order transition or crossover region. In the vicinity of the critical point, the peak of the $\chi_{\ell}$ rapidly diminishes. We have found two qualitative differences between the Weiss and Haar measure MFA on the 
Polyakov loop susceptibilities: First, the peak of $\chi_{e}$ is more strongly locked to the chiral phase boundary in Weiss MFA than the Haar measure MFA case. Second, the $Z_{3}$ deconfinement dynamics artificially remains in the Haar measure MFA and disappears by taking account of the Polyakov loop fluctuations in Weiss MFA. Our findings are summarized in Fig. 5 (upper, Haar measure MFA result) and Fig. 8 (Weiss MFA result). The above difference results from the fact that the effective potential of Weiss MFA does not admit any remnant of the $Z_{3}$ symmetric structure in sharp contrast to the Haar measure MFA and many other chiral effective models $[42,43,45]$. Thus, the Weiss MFA does not support the isolated deconfinement transition/crossover from the chiral phase boundary at large $\mu$.

There are several future directions to be investigated. First, it is important to evaluate the higher order terms of the strong-coupling expansion, and/or to invent a resummation technique to account for the higher orders. From this viewpoint, we find recent developments for the Polyakov loop effective potential [52]. Second, it is desirable to establish the exact evaluation of each order of the strongcoupling expansion beyond the mean-field approximation and $1 / d$ expansion. This will be achieved by extending the MDP works [31,32] to include the higher-order of expansions as well as the Polyakov loop effects. Another method to go beyond MFA is the Monte-Carlo simulations for the auxiliary field integrals at each order of the expansion [33]. Third, it is interesting to evaluate the complex phase effect of Polyakov loops; The susceptibilities associated with the phase may give a new probe of the QCD phase transition [53]. And finally, the Weiss MFA results, especially the quark and Polyakov loop thermal excitations summarized in Table $\mathrm{V}$, may open a possibility to invent an upgraded version of the PNJL-type model which more reasonably describes the interplay between the chiral and deconfinement dynamics.

\section{ACKNOWLEDGMENTS}

We thank Maria Paola Lombardo and Philippe de Forcrand for fruitful discussions. This work was supported in part by the Grants-in-Aid for Scientific Research from Japan Society for the Promotion of Science (JSPS) (Nos. 22-3314, 15K05079, 15H03663, 16K05350), for Young Scientists (B) No. 15K17644 (Kohtaroh Miura), the Grants-in-Aid for Scientific Research on Innovative Areas from Ministry of Education, Culture, Sports, Science and Technology (MEXT) (Nos. 24105001, 24105008), and the Yukawa International Program for Quark-hadron Sciences (YIPQS). Kohtaroh Miura was supported by in part by the OCEVU Laboratoire d'excellence (ANR-11LABX-0060) the A*MIDEX Project (ANR-11-IDEX0001-02), which are funded by the "Investissements d'Avenir" French government program and managed by the "Agence nationale de la recherche" (ANR).

\section{APPENDIX: EFFECTIVE POTENTIAL IN STRONG-COUPLING LATTICE QCD}

We briefly review the derivation of the effective potential Eq. (1) based on our previous papers [27,28]. We start from the lattice QCD action with one species of staggered fermion $(\chi)$ with a current quark mass $\left(m_{0}\right)$ and chemical potential $(\mu)$,

$$
\begin{aligned}
\mathcal{Z}_{\mathrm{LQCD}} & =\int \mathcal{D}\left[\chi, \bar{\chi}, U_{\nu}\right] e^{-S_{\mathrm{LQCD}}\left[\chi, \bar{\chi}, U_{\nu}\right]}, \\
S_{\mathrm{LQCD}} & =S_{F}+S_{G}+m_{0} \sum_{x} \bar{\chi}_{x} \chi_{x},
\end{aligned}
$$

where,

$$
\begin{aligned}
S_{F} & =\frac{1}{2} \sum_{\nu, x}\left[\eta_{\nu, x} \bar{\chi}_{x} U_{\nu, x} \chi_{x+\hat{\nu}}-\eta_{\nu, x}^{-1}(\text { H.c. })\right], \\
\eta_{\nu, x} & =\exp \left(\mu \delta_{\nu 0}\right)(-1)^{x_{0}+\cdots+x_{\nu-1}} \\
S_{G} & =\beta \sum_{P}\left[1-\frac{1}{2 N_{c}}\left[U_{P}+U_{P}^{\dagger}\right]\right] .
\end{aligned}
$$

We have employed lattice units $a=1$. The $U_{\nu, x} \in S U\left(N_{c}\right)$ and $U_{P=\mu \nu, x}=\operatorname{tr}_{c}\left[U_{\mu, x} U_{\nu, x+\hat{\mu}} U_{\mu, x+\hat{\nu}}^{\dagger} U_{\nu, x}^{\dagger}\right]$ represent the link- and plaquette-variable, respectively. In the chiral limit $\left(m_{0} \rightarrow 0\right)$, the action has the $U_{\chi}(1)$ chiral symmetry, which is enhanced to $S U\left(N_{f}=4\right)$ in the continuum limit.

There are four main steps to derive the effective potential from the lattice QCD action (A1) [28]: First, we carry out the strong-coupling expansion, and integrate out the spatial link variables in each order. The effective action is obtained as a function of various hadronic composites. For the composites including the staggered quarks $(\chi, \bar{\chi})$, we take account of the terms up to $\mathcal{O}\left(1 / g^{6}\right)$, and extract from them the leading order terms of the $1 / d$ expansion $\mathcal{O}\left(1 / d^{0}\right)$ [54]. For the pure gluonic composites, we take account of the leading order contributions to the Polyakov-loop $\left[\mathcal{O}\left(1 / g^{2 N_{\tau}}\right), N_{\tau}\right.$ : lattice temporal extension]. The hadronic composites are summarized in Table I, and the effective action is expressed by using these composites,

$$
S_{\mathrm{eff}}=S_{\mathrm{eff}}^{\mathrm{NNO}}+S_{\mathrm{eff}}^{\mathrm{Pol}},
$$

TABLE I. The hadronic composites which appears after the spatial link integrals.

\begin{tabular}{lc}
\hline \hline Symbol & Composites \\
\hline$M_{x}$ & $\bar{\chi}_{x} \chi_{x}$ \\
$\left(V_{x}^{+}, V_{x}^{-}\right)$ & $\left(\bar{\chi}_{x} e^{\mu} U_{0, x} \chi_{x+\hat{0}}, \bar{\chi}_{x+\hat{0}} e^{-\mu} U_{0, x}^{\dagger} \chi_{x}\right)$ \\
$\left(W_{x}^{+}, W_{x}^{-}\right)$ & $\left(\bar{\chi}_{x} e^{2 \mu} U_{0, x} U_{0, x+\hat{0}} \chi_{x+2 \hat{0}}, \bar{\chi}_{x+2 \hat{0}} e^{-2 \mu} U_{0, x+\hat{0}}^{\dagger} U_{0, x}^{\dagger} \chi_{x}\right)$ \\
$L_{p, \mathbf{x}}$ & $\operatorname{tr}_{c}\left[\prod_{\tau} U_{0, \mathbf{x} \tau}\right] / N_{c}$ \\
\hline \hline
\end{tabular}


TABLE II. The auxiliary field $\Phi$ and $(\ell, \bar{\ell})$ See also Table I.

\begin{tabular}{lc}
\hline \hline Symbol & Mean Fields Contents \\
\hline$\sigma$ & $-\langle M\rangle$ \\
$\left(\bar{\psi}_{\tau \tau}, \psi_{\tau \tau}\right)$ & $\left(\left\langle W^{+}\right\rangle,\left\langle W^{-}\right\rangle\right)$ \\
$\left(\bar{\psi}_{s s}, \psi_{s s}\right)$ & $(\langle M M\rangle,\langle M M M M\rangle)$ \\
$\left(\bar{\psi}_{\tau s}, \psi_{\tau s}\right)$ & $\left(-\left\langle V^{+} V^{-}\right\rangle, 2\langle M M\rangle\right)$ \\
$\left(\bar{\psi}_{\tau}, \psi_{\tau}\right)$ & $\left(-\left\langle V^{+}\right\rangle,\left\langle V^{-}\right\rangle\right)$ \\
$\left(\bar{\psi}_{s}, \psi_{s}\right)$ & $(\langle M M\rangle,\langle M M\rangle)$ \\
$(\ell, \bar{\ell})$ & $\left(\left\langle L_{p}\right\rangle,\left\langle\bar{L}_{p}\right\rangle\right)$ \\
\hline \hline
\end{tabular}

with

$S_{\text {eff }}^{\text {Pol }}=-N_{c}^{2}\left(\frac{1}{g^{2} N_{c}}\right)^{N_{\tau}=1 / T} \sum_{j, \mathbf{x}}\left[\bar{L}_{p, \mathbf{x}} L_{p, \mathbf{x}+\hat{j}}+\right.$ H.c. $]$,

and

$$
\begin{aligned}
S_{\mathrm{eff}}^{\mathrm{NNLO}}= & \sum_{x} \frac{1}{2}\left(V_{x}^{+}-V_{x}^{-}\right)+\sum_{x, j>0}\left[-\frac{b_{\sigma}}{2 d}[M M]_{j, x}\right. \\
& +\frac{\beta_{\tau}}{4 d}\left[V^{+} V^{-}+V^{-} V^{+}\right]_{j, x}-\sum_{k>0, k \neq j} \frac{\beta_{s}[M M M M]_{j k, x}}{2 d(d-1)} \\
& -\frac{\beta_{\tau \tau}}{2 d}\left[W^{+} W^{-}+W^{-} W^{+}\right]_{j, x} \\
& +\sum_{|k| \neq j}\left[\sum_{\substack{|k|,|l||0| \\
|l| j|l| \neq|<|}} \frac{-\beta_{s s}[M M M M]_{j k, x}[M M]_{j, x+\hat{l}}}{4 d(d-1)(d-2)}\right. \\
& +\frac{\beta_{\tau s}\left[V^{+} V^{-}+V^{-} V^{+}\right]_{j, x}}{8 d(d-1)} \\
& \left.\times\left([M M]_{j, x+\hat{k}}+[M M]_{j, x+\hat{k}+\hat{0})}\right)\right] .
\end{aligned}
$$

We have introduced a short-hand notation

$$
\begin{aligned}
{[A B]_{j, x} } & =A_{x} B_{x+\hat{j}}, \\
{[A B C D]_{j k, x} } & =A_{x} B_{x+\hat{j}} C_{x+\hat{j}+\hat{k}} D_{x+\hat{k}},
\end{aligned}
$$

and the couplings $\beta \ldots$ in Eq. (A8) are summarized in Table III.

Second, we introduce the auxiliary fields for the hadronic composites to bosonize the effective action $S_{\mathrm{eff}}^{\mathrm{NNLO}}$, and perform the static mean-field and saddle-point approximations. The auxiliary fields are summarized in Table II, and the $S_{\mathrm{eff}}^{\mathrm{NNOO}}$ reduces into

$$
S_{\mathrm{eff}}^{\mathrm{NNLO}} \simeq S_{\mathrm{eff}}^{\mathrm{F}}+S_{\mathrm{eff}}^{\mathrm{X}}
$$

where
TABLE III. The coupling coefficients appearing in the effective action/potential. Here, $g, N_{c}=3$, and $d=3$ represents the gauge coupling, number of color, and spatial dimension, respectively. See Table II for the auxiliary fields $\left(\psi_{\ldots}, \bar{\psi}_{\ldots}\right)$.

\begin{tabular}{lc}
\hline \hline Symbol & Definition \\
\hline$b_{\sigma}$ & $d /\left(2 N_{c}\right)$ \\
$\beta_{t}$ & $\left(d /\left(N_{c}^{2} g^{2}\right)\right) \cdot\left(1+1 /\left(2 g^{2}\right)\right)$ \\
$\beta_{s}$ & $\left(d(d-1) /\left(8 N_{c}^{4} g^{2}\right)\right) \cdot\left(1+1 /\left(2 g^{2}\right)\right)$ \\
$b_{\sigma}^{\prime}$ & $b_{\sigma}+2\left[\beta_{s s} \psi_{s s}+\beta_{\tau s} \bar{\psi}_{\tau s}+\beta_{s}^{\prime}\left(\psi_{s}+\bar{\psi}_{s}\right)\right]$ \\
$\beta_{t}^{\prime}$ & $\beta_{t}+\beta_{\tau s} \psi_{\tau s}$ \\
$\beta_{s}^{\prime}$ & $\beta_{s}+2 \beta_{s s} \bar{\psi}_{s s}$ \\
$\beta_{\tau \tau}$ & $d /\left(2 N_{c}^{3} g^{4}\right)$ \\
$\beta_{s s}$ & $d(d-1)(d-2) /\left(16 N_{c}^{7} g^{4}\right)$ \\
$\beta_{\tau s}$ & $d(d-1) /\left(2 N_{c}^{5} g^{4}\right)$ \\
\hline \hline
\end{tabular}

$S_{\mathrm{eff}}^{\mathrm{F}}=\sqrt{Z_{+} Z_{-}} \sum_{x y} \bar{\chi}_{x} G_{x y}^{-1}\left(\tilde{m}_{q}, \tilde{\mu}\right) \chi_{y}$,

$G_{x y}^{-1}\left(\tilde{m}_{q}, \tilde{\mu}\right)=\tilde{m}_{q} \delta_{x y}+\frac{\delta_{\mathbf{x y}}}{2}\left[e^{\tilde{\mu}} U_{0, x} \delta_{x+\hat{0}, y}-e^{-\tilde{\mu}} U_{0, x}^{\dagger} \delta_{x-\hat{0}, y}\right]$,

$$
\begin{aligned}
S_{\mathrm{eff}}^{\mathrm{X}}= & N_{\tau} N_{s}^{d}\left[b_{\sigma}^{\prime} \sigma^{2}+\frac{1}{2} \beta_{\tau}^{\prime} \bar{\psi}_{\tau} \psi_{\tau}+\frac{1}{2} \beta_{s}^{\prime} \bar{\psi}_{s} \psi_{s}\right. \\
& \left.+\beta_{\tau \tau} \bar{\psi}_{\tau \tau} \psi_{\tau \tau}+\beta_{s s} \bar{\psi}_{s s} \psi_{s s}+\frac{1}{2} \beta_{\tau s} \bar{\psi}_{\tau s} \psi_{\tau s}\right] .
\end{aligned}
$$

Here, the dynamical quark mass $\tilde{m}_{q}$, the shifted quark chemical potential $\tilde{\mu}$, and the wave function renormalization factor $\sqrt{Z_{+} Z_{-}}$are summarized in Table IV, and the $N_{t(s)}$ represents the temporal (spatial) lattice extension.

Third, we carry out the Gaussian integral over the staggered quarks $(\chi, \bar{\chi})$ in Eq. (A12) in the antiperiodic boundary condition. The resultant quark determinant at finite $T$ is then calculated by using the Matsubara method in the Polyakov gauge for temporal link variables [41],

TABLE IV. Quantities which govern the property of the effective potential. See Table III for the couplings $\left(b_{\sigma}^{\prime}, \beta_{\tau}^{\prime}, \beta_{\tau \tau}\right)$ and Table II for the auxiliary fields $\left(\sigma, \psi_{\tau}, \bar{\psi}_{\tau}, \psi_{\tau \tau}, \bar{\psi}_{\tau \tau}\right)$.

\begin{tabular}{lcc}
\hline \hline Symbol & Definition & Meanings \\
\hline$\tilde{m}_{q}$ & $m_{q}^{\prime} / \sqrt{Z_{+} Z_{-}}$ & Dynamical quark mass \\
& $m_{q}^{\prime}=b_{\sigma}^{\prime} \sigma+m_{0}$ & \\
& $-\beta_{\tau \tau}\left(\bar{\psi}_{\tau \tau}+\psi_{\tau \tau}\right)$ & \\
$\sqrt{Z_{+} Z_{-}}$ & $Z_{+}=1+\beta_{\tau}^{\prime} \bar{\psi}_{\tau}$ & Wave function \\
& $+4 \beta_{\tau \tau} m_{q}^{\prime} \bar{\psi}_{\tau \tau}$ & Renormalization factor \\
& $Z_{-}=1+\beta_{\tau}^{\prime} \psi_{\tau}$ & \\
& $+4 \beta_{\tau \tau} m_{q}^{\prime} \psi_{\tau \tau}$ & \\
$E_{q}$ & $\sinh ^{-1} \tilde{m}_{q}$ & Quark excitation energy \\
$\tilde{\mu}$ & $\mu-\log \sqrt{Z_{+} / Z_{-}}$ & Shifted chemical potential \\
\hline \hline
\end{tabular}




$$
\begin{aligned}
\int \mathrm{D}[\chi, \bar{\chi}] e^{-S_{\text {eff }}^{\mathrm{F}}}= & \prod_{\mathbf{x}}\left[e^{N_{c}\left(\log \sqrt{Z_{+} Z_{-}}+E_{q}\right) / T}\right. \\
& \times \operatorname{det}_{c}\left[\left(1+N_{c} L_{p, \mathbf{x}} e^{-\left(E_{q}-\tilde{\mu}\right) / T}\right)\right. \\
& \left.\left.\times\left(1+N_{c} \bar{L}_{p, \mathbf{x}} e^{-\left(E_{q}+\tilde{\mu}\right) / T}\right)\right]\right],
\end{aligned}
$$

with $E_{q}=\sinh ^{-1} \tilde{m}_{q}$. Temperature $T$ is now considered as a continuous valued number (see the Appendix in Ref. [24] for details). The Polyakov loop $L_{p, \mathbf{x}}$ has appeared in the determinant via the quark hopping wrapping around the temporal direction in addition to the Plaquette effects Eq. (A7).

Finally, we evaluate the $L_{p, \mathbf{x}}$ effects in the path integral over the temporal link variable $U_{0}$ in two approximation schemes: Haar measure and Weiss MFA. In the former, we replace the Polyakov loop $L_{p, \mathbf{x}}$ contained in Eq. (A7) and (A15) as well as the Haar measure of the $U_{0}$ path integral with a constant mean-field $(\ell, \bar{\ell})$ instead of performing the $U_{0}$ path integral. In the latter, we introduce a meanfield $(\ell, \bar{\ell})$ via the extended Hubbard-Stratonovich transformation [25] in Eq. (A7), and exactly carry out the $U_{0}$ path integral to include the fluctuation effects from $(\ell, \bar{\ell})$ [28].

As a result, we obtain the effective potential

$$
\begin{aligned}
\mathcal{F}_{\text {eff }}^{\mathrm{H} / \mathrm{W}} & \left(\Phi, \ell, \bar{\ell} ; \beta, m_{0}, T, \mu\right) \\
= & \mathcal{F}_{\mathrm{X}}(\Phi, \beta)+\mathcal{F}_{\text {det }}^{\mathrm{H} / \mathrm{W}}\left(\Phi, \beta, m_{0}, T, \mu\right) \\
& +\mathcal{F}_{\mathrm{P}}^{\mathrm{H} / \mathrm{w}}(\ell, \bar{\ell}, \beta, T)+\mathcal{O}\left(1 / g^{6}, 1 / \sqrt{d}\right) .
\end{aligned}
$$

The auxiliary field term is given by Eq. (A14) and is common in both Haar measure MFA and Weiss MFA,

$$
\mathcal{F}_{\mathrm{X}}(\Phi, \beta)=S_{\text {eff }}^{\mathrm{X}} /\left(N_{t} N_{s}^{3}\right) .
$$

The quark determinant and the Polyakov loop effects are given as

$$
\begin{aligned}
\mathcal{F}_{\mathrm{det}}^{\mathrm{H}}= & -N_{c} E_{q}-N_{c} \log \sqrt{Z_{+} Z_{-}} \\
& -T\left(\log \mathcal{R}_{q}\left(E_{q}-\tilde{\mu}, \ell, \bar{\ell}\right)+\log \mathcal{R}_{q}\left(E_{q}+\tilde{\mu}, \bar{\ell}, \ell\right)\right),
\end{aligned}
$$

$$
\begin{aligned}
\mathcal{R}_{q}(x, y, \bar{y}) & \equiv 1+N_{c}\left(y e^{-x / T}+\bar{y} e^{-2 x / T}\right)+e^{-3 x / T} \\
\mathcal{F}_{\mathrm{P}}^{\mathrm{H}} & =-2 T d N_{c}^{2}\left(\frac{1}{g^{2} N_{c}}\right)^{1 / T} \bar{\ell} \ell-T \log \mathcal{R}_{\text {Haar }}(\ell, \bar{\ell}),
\end{aligned}
$$

$$
\mathcal{R}_{\text {Haar }}(\ell, \bar{\ell}) \equiv 1-6 \bar{\ell} \ell-3(\bar{\ell} \ell)^{2}+4\left(\ell^{N_{c}}+\bar{\ell}^{N_{c}}\right),
$$

in Haar measure MFA case, and

$$
\begin{aligned}
\mathcal{F}_{\mathrm{P}}^{\mathrm{W}}+\mathcal{F}_{\mathrm{det}}^{\mathrm{W}}= & 2 T d N_{c}^{2}\left(\frac{1}{g^{2} N_{c}}\right)^{1 / T} \bar{\ell} \ell \\
& -T \log \left[\sum_{I} \mathcal{Q}^{I}(\Phi) \mathcal{P}^{I}(\ell, \bar{\ell})\right],
\end{aligned}
$$

$\mathcal{P}^{I}(\ell, \bar{\ell})=\sum_{n=-\infty}^{\infty}(\sqrt{\ell / \bar{\ell}})^{-N_{c} n+N_{\mathrm{Q}}^{I}} \mathcal{P}_{n}^{I}(\sqrt{\ell \bar{\ell}})$,

in Weiss MFA case. In Eqs. (A21) and (A22), the index I labels a pattern of thermal excitations of the quark composites, and the fermionic thermal excitation effects $\mathcal{Q}^{I}$, the Polyakov loop thermal excitation effects $\mathcal{P}_{n}^{I}$, and the quark number index $N_{\mathrm{Q}}^{I}$ are summarized in Table $\mathrm{V}$.

As indicated in Eq. (A19) and (A21), the $Z_{3}$ symmetric term remains in the Haar measure MFA, but not in the Weiss MFA up to the first $\bar{\ell} \ell$ term. In the latter, the path integral over the temporal link variable $U_{0}$ which accounts for the summation over the Polyakov loop fluctuations spoils the $Z_{3}$ symmetry in the presence of the dynamical quarks. In heavy quark mass limit $m_{0} \rightarrow \infty$, the $Z_{3}$ symmetry recovers in the Weiss MFA as follows: In the effective potential of Weiss MFA, the factor $(\sqrt{\ell / \bar{\ell}})^{-N_{c} n+N_{\mathrm{Q}}^{I}}$ in Eq. (A22) gives a unique source of the explicit $Z_{3}$ symmetry breaking $\left((\ell, \bar{\ell}) \rightarrow\left(\Omega \ell, \Omega^{-1} \bar{\ell}\right)\right.$, $\left.\Omega \in Z_{3}\right)$. For $m_{0} \rightarrow \infty$ or equivalently $E_{q} \gg T, \mu$, the three mesonic thermal excitation $\mathcal{Q}^{I=\text { MMM }}$ in Table $\mathrm{V}$ becomes dominant, and it does not carry the quark number $N_{\mathrm{Q}}^{I=\mathrm{MMM}}=0$. Therefore, the Eq. (11) reduces to

$$
\begin{aligned}
\mathcal{F}_{\mathrm{P}}^{\mathrm{W}}+\mathcal{F}_{\mathrm{det}}^{\mathrm{W}}= & 2 T d N_{c}^{2}\left(\frac{1}{g^{2} N_{c}}\right)^{1 / T} \bar{\ell} \ell-T \log \left[\mathcal{Q}^{I=\mathrm{MMM}}(\Phi)\right. \\
& \left.\times \sum_{n=-\infty}^{\infty}(\sqrt{\ell / \bar{\ell}})^{-N_{c} n} \mathcal{P}_{n}^{I=\mathrm{MMM}}(\sqrt{\ell \bar{\ell}})\right]
\end{aligned}
$$

This expression is invariant under the $Z_{3}$ transformation, $(\ell, \bar{\ell}) \rightarrow\left(\Omega \ell, \Omega^{-1} \bar{\ell}\right)$ with the property $\Omega^{N_{c} n}=\mathbf{1}$ for $N_{c}=3$.

Finally, we consider the confinement limit $(\ell, \bar{\ell} \rightarrow 0)$ in the Weiss MFA. The quark determinant effect (A21) includes the Polyakov loop thermal excitation $\mathcal{P}_{n}^{I}$, which are solely characterized by the nth-order modified Bessel functions as shown in Table V. In the limit $(\ell, \bar{\ell} \rightarrow 0)$, the 0th-order modified Bessel function remains finite $\left(I_{0}(x \rightarrow 0)=1\right)$ while the others vanish $\left(I_{n \neq 0}(x \rightarrow 0)=0\right)$. Consequently, the only thermal excitations which carry the quark number 0 and \pm 3 survives in Table $\mathrm{V}$, and the effective potential reduces into the one which we have derived in our previous work [25],

$$
\begin{aligned}
\mathcal{F}_{\mathrm{eff}}^{\mathrm{W}} & \left.\left(\Phi, \ell, \bar{\ell} ; \beta, m_{0}, T, \mu\right)\right|_{\ell, \bar{\ell}=0} \\
& \rightarrow \mathcal{F}_{\mathrm{eff}}^{\mathrm{NLO}}\left(\Phi ; \beta, m_{0}, T, \mu\right)=\mathcal{F}_{\mathrm{X}}(\Phi, \beta) \\
& -T \log \left[\left(2 \cosh \frac{E_{q}}{T}\right)^{N_{c}}-4 \cosh \frac{E_{q}}{T}+2 \cosh \frac{N_{c} \tilde{\mu}}{T}\right] .
\end{aligned}
$$


TABLE V. The thermal excitation effects $\mathcal{P}_{n}^{I}$ and $\mathcal{Q}^{I}$ in the quark determinant of the Weiss MFA, Eq. (A21). The left column represents the excitation channel with the label $I$ in the text: (M, B, Q, D) stands for (mesonic, baryonic, quark, diquark) excitation. The quark excitation energy $E_{q}$ and modified chemical potential $\tilde{\mu}$ appearing in the third column are explained in Table IV. In the right column, $I_{n}$ represents a modified Bessel function with the argument $x=4 d N_{c}\left(\beta /\left(2 N_{c}^{2}\right)\right)^{1 / T} \sqrt{\ell \bar{\ell}}$.

\begin{tabular}{|c|c|c|c|}
\hline Excitation $(I)$ & $N_{\mathrm{Q}}^{I}$ & $\mathcal{Q}^{I}(\Phi)$ & $\mathcal{P}_{n}^{I}(\sqrt{\ell \bar{\ell}})$ \\
\hline MMM & 0 & $\left(2 \cosh \left(E_{q} / T\right)\right)^{N_{c}}$ & $\mathcal{P}_{n}^{\mathrm{MMM}}=I_{n}^{3}-I_{n-2} I_{n} I_{n+2}-2 I_{n-1} I_{n} I_{n+1}+I_{n-2} I_{n+1}^{2}+I_{n-1}^{2} I_{n+2}$ \\
\hline MQQ̄ & 0 & $2 \cosh \left(E_{q} / T\right)$ & $\begin{aligned} \mathcal{P}_{n}^{\mathrm{MQQ}}= & -2\left(I_{n}^{3}-I_{n-2} I_{n} I_{n+2}\right)+5 I_{n-1} I_{n} I_{n+1}-3\left(I_{n-2} I_{n+1}^{2}+I_{n-1}^{2} I_{n+2}\right) \\
& -I_{n-3} I_{n} I_{n+3}+I_{n-1} I_{n-2} I_{n+3}+I_{n-3} I_{n+1} I_{n+2}\end{aligned}$ \\
\hline $\mathrm{B}$ & 3 & $e^{N_{c} \tilde{\mu} / T}$ & $\mathcal{P}_{n}^{\mathrm{B}}=\mathcal{P}_{n-1}^{\mathrm{MMM}}$ \\
\hline$\overline{\mathrm{B}}$ & -3 & $e^{-N_{c} \tilde{\mu} / T}$ & $\mathcal{P}_{n}^{\overline{\mathrm{B}}}=\mathcal{P}_{n+1}^{\mathrm{MMM}}$ \\
\hline MMQ & 1 & $e^{\tilde{\mu} / T}\left(2 \cosh \left(E_{q} / T\right)\right)^{2}$ & $\mathcal{P}_{n}^{\mathrm{MMQ}}=I_{n-1} I_{n}^{2}+I_{n-3} I_{n+1}^{2}-I_{n-1}^{2} I_{n+1}+I_{n-2} I_{n-1} I_{n+2}-I_{n-3} I_{n} I_{n+2}-I_{n-2} I_{n} I_{n+1}$ \\
\hline MMQ̄ & -1 & $e^{-\tilde{\mu} / T}\left(2 \cosh \left(E_{q} / T\right)\right)^{2}$ & $\mathcal{P}_{n}^{\mathrm{MMQ}}=I_{n+1} I_{n}^{2}+I_{n+3} I_{n-1}^{2}-I_{n+1}^{2} I_{n-1}+I_{n-2} I_{n+1} I_{n+2}-I_{n+3} I_{n} I_{n-2}-I_{n+2} I_{n} I_{n-1}$ \\
\hline MD & 2 & $e^{2 \tilde{\mu} / T} 2 \cosh \left(E_{q} / T\right)$ & $\mathcal{P}_{n}^{\mathrm{MD}}=\mathcal{P}_{n-1}^{\mathrm{MMQ}}$ \\
\hline MD̄ & -2 & $e^{-2 \tilde{\mu} / T} 2 \cosh \left(E_{q} / T\right)$ & $\mathcal{P}_{n}^{\mathrm{M} \overline{\mathrm{D}}}=\mathcal{P}_{n+1}^{\mathrm{MMQ}}$ \\
\hline $\mathrm{DQ}$ & 1 & $e^{\tilde{\mu} / T}$ & $\begin{aligned} \mathcal{P}_{n}^{\mathrm{D} \overline{\mathrm{Q}}}= & 2\left(I_{n-1}^{2} I_{n+1}-I_{n-2} I_{n-1} I_{n+2}+I_{n-3} I_{n} I_{n+2}\right) \\
& +I_{n-2}^{2} I_{n+3}-I_{n-1} I_{n}^{2}-I_{n-3} I_{n+1}^{2}-I_{n-3} I_{n-1} I_{n+3}\end{aligned}$ \\
\hline QD̄ & -1 & $e^{-\tilde{\mu} / T}$ & $\begin{aligned} & \mathcal{P}_{n}^{\mathrm{Q} \overline{\mathrm{D}}=} 2\left(I_{n+1}^{2} I_{n-1}-I_{n-2} I_{n+1} I_{n+2}+I_{n-2} I_{n} I_{n+3}\right) \\
&+I_{n+2}^{2} I_{n-3}-I_{n+1} I_{n}^{2}-I_{n+3} I_{n-1}^{2}-I_{n-3} I_{n+1} I_{n+3} \\
&\end{aligned}$ \\
\hline
\end{tabular}

[1] For a recent lattice review of the QCD at finite temperature and/or density, see O. Philipsen, The QCD equation of state from the lattice, Prog. Part. Nucl. Phys. 70, 55 (2013).

[2] For a recent review of the QCD phase diagram, see K. Fukushima and C. Sasaki, The phase diagram of nuclear and quark matter at high baryon density, Prog. Part. Nucl. Phys. 72, 99 (2013).

[3] For recent results and reviews, see, S. Borsanyi, Z. Fodor, C. Hoelbling, S. D. Katz, S. Krieg, C. Ratti, and K. K. Szabo (Wuppertal-Budapest Collaboration), Is there still any Tc mystery in lattice QCD results with physical masses in the continuum limit III, J. High Energy Phys. 09 (2010) 073.

[4] D. Bailin and A. Love, Superfluidity and superconductivity in relativistic fermion systems, Phys. Rep. 107, 325 (1984); R. Rapp, T. Schafer, E. V. Shuryak, and M. Velkovsky, Diquark Bose Condensates in High Density Matter and Instantons, Phys. Rev. Lett. 81, 53 (1998); M. G. Alford, K. Rajagopal, and F. Wilczek, QCD at finite baryon density: Nucleon droplets and color superconductivity, Phys. Lett. B 422, 247 (1998); M. G. Alford, Color superconducting quark matter, Annu. Rev. Nucl. Part. Sci. 51, 131 (2001); D. T. Son, Superconductivity by long-range color magnetic interaction in high-density quark matter, Phys. Rev. D 59, 094019 (1999); M. G. Alford, K. Rajagopal, and F. Wilczek, Color-flavor locking and chiral symmetry breaking in high density QCD, Nucl. Phys. B537, 443 (1999); J. Berges and K. Rajagopal, Color superconductivity and chiral symmetry restoration at nonzero baryon density and temperature, Nucl. Phys. B538, 215 (1999); K. Iida and G. Baym,
The superfluid phases of quark matter: Ginzburg-Landau theory and color neutrality, Phys. Rev. D 63, 074018 (2001); Erratum, Phys. Rev. D 66, 059903(E) (2002); M. Iwasaki and T. Iwado, Superconductivity in the quark matter, Phys. Lett. B 350, 163 (1995).

[5] L. McLerran and R. D. Pisarski, Phases of cold, dense quarks at large $N_{c}$, Nucl. Phys. A796, 83 (2007).

[6] Y. Hidaka, L. D. McLerran, and R. D. Pisarski, Baryons and the phase diagram for a large number of colors and flavors, Nucl. Phys. A808, 117 (2008); L. McLerran, K. Redlich, and C. Sasaki, Quarkyonic matter and chiral symmetry breaking, Nucl. Phys. A824, 86 (2009).

[7] L. McLerran, Quarkyonic matter and the revised phase diagram of QCD, Nucl. Phys. A830, 709C (2009).

[8] T. Kojo, Y. Hidaka, K. Fukushima, L. D. McLerran, and R. D. Pisarski, Interweaving chiral spirals, Nucl. Phys. A875, 94 (2012); T. Kojo, Y. Hidaka, L. McLerran, and R. D. Pisarski, Quarkyonic chiral spirals, Nucl. Phys. A843, 37 (2010).

[9] For a recent review, see, C. Gale, S. Jeon, and B. Schenke, Hydrodynamic modeling of heavy-ion collisions, Int. J. Mod. Phys. A 28, 1340011 (2013).

[10] M. Asakawa and K. Yazaki, Chiral restoration at finite density and temperature, Nucl. Phys. A504, 668 (1989); M. A. Stephanov, K. Rajagopal, and E. V. Shuryak, Signatures of the Tricritical Point in QCD, Phys. Rev. Lett. 81, 4816 (1998).

[11] B. Mohanty (STAR Collaboration), STAR experiment results from the beam energy scan program at RHIC, J. Phys. G 38, 124023 (2011). 
[12] For a review of a finite chemical potential on lattice, see $S$. Muroya, A. Nakamura, C. Nonaka, and T. Takaishi, Lattice QCD at finite density: An introductory review, Prog. Theor. Phys. 110, 615 (2003).

[13] G. Aarts, Complex Langevin dynamics and other approaches at finite chemical potential, Proc. Sci., LATTICE2012 (2012) 017 [arXiv:1302.3028].

[14] H. Fujii, D. Honda, M. Kato, Y. Kikukawa, S. Komatsu, and T. Sano, Hybrid Monte Carlo on Lefschetz thimbles-A study of the residual sign problem, J. High Energy Phys. 10 (2013) 147.

[15] M. Cristoforett, F. Di Renzo, and L. Scorzato (AuroraScience Collaboration), New approach to the sign problem in quantum field theories: High density QCD on a Lefschetz thimble, Phys. Rev. D 86, 074506 (2012).

[16] The review of the pioneering works for the strong-coupling expansion is found in the text book, I. Montvay and G. Münster, Quantum Fields on a Lattice (Cambridge University Press, Cambridge, England, 1994); M. Creutz, Quarks, Gluons, and Lattices (Cambridge University Press, Cambridge, England, 1983).

[17] K. G. Wilson, Confinement of quarks, Phys. Rev. D 10, 2445 (1974).

[18] M. Creutz, Monte Carlo study of quantized SU(2) gauge theory, Phys. Rev. D 21, 2308 (1980); M. Creutz and K. J. M. Moriarty, Numerical studies of Wilson loops in SU(3) gauge theory in four-dimensions, Phys. Rev. D 26, 2166 (1982).

[19] G. Münster, High temperature expansions for the free energy of vortices, respectively the string tension in lattice gauge theories, Nucl. Phys. B180, 23 (1981).

[20] K. Fukushima, Toward understanding the lattice QCD results from the strong coupling analysis, Prog. Theor. Phys. Suppl. 153, 204 (2004).

[21] Y. Nishida, K. Fukushima, and T. Hatsuda, Thermodynamics of strong coupling 2-color QCD with chiral and diquark condensates, Phys. Rep. 398, 281 (2004).

[22] Y. Nishida, Phase structures of strong coupling lattice QCD with finite baryon and isospin density, Phys. Rev. D 69 , 094501 (2004).

[23] V. Azcoiti, G. Di Carlo, A. Galante, and V. Laliena, Diquark condensation at strong coupling, J. High Energy Phys. 09 (2003) 014.

[24] N. Kawamoto, K. Miura, A. Ohnishi, and T. Ohnuma, Phase diagram at finite temperature and quark density in the strong coupling limit of lattice QCD for color SU(3), Phys. Rev. D 75, 014502 (2007).

[25] K. Miura, T.Z. Nakano, A. Ohnishi, and N. Kawamoto, Phase diagram evolution at finite coupling in strong coupling lattice QCD, Phys. Rev. D 80, 074034 (2009); Quarkyonic phase in lattice QCD at strong coupling, Prog. Theor. Phys. 122, 1045 (2009).

[26] K. Miura, T.Z. Nakano, A. Ohnishi, and N. Kawamoto, Strong-coupling lattice study for QCD phase diagram including both chiral and deconfinement dynamics, arXiv:1106.1219.

[27] T. Z. Nakano, K. Miura, and A. Ohnishi, Effective potential in the strong-coupling lattice QCD with next-to-next-toleading order effects, Prog. Theor. Phys. 123, 825 (2010).
[28] T.Z. Nakano, K. Miura, and A. Ohnishi, Chiral and deconfinement transitions in strong coupling lattice QCD with finite coupling and Polyakov-loop effects, Phys. Rev. D 83, 016014 (2011).

[29] B. Bringoltz, Chiral crystals in strong-coupling lattice QCD at nonzero chemical potential, J. High Energy Phys. 03 (2007) 016.

[30] P. de Forcrand and M. Fromm, Nuclear Physics from Lattice QCD at Strong Coupling, Phys. Rev. Lett. 104, 112005 (2010).

[31] P. de Forcrand, J. Langelage, O. Philipsen, and W. Unger, The Lattice QCD Phase Diagram in and away from the Strong Coupling Limit, Phys. Rev. Lett. 113, 152002 (2014).

[32] P. de Forcrand, M. Fromm, J. Langelage, K. Miura, O. Philipsen, and W. Unger, Towards corrections to the strong coupling limit of staggered lattice QCD, Proc. Sci., LATTICE2011 (2011) 212 [arXiv:1111.4677]; P. de Forcrand, J. Langelage, O. Philipsen, and W. Unger, The phase diagram of strong coupling QCD including gauge corrections, Proc. Sci., LATTICE2013 (2014) 142 [arXiv:1312.0589].

[33] T. Ichihara, A. Ohnishi, and T. Z. Nakano, Auxiliary field Monte-Carlo simulation of strong coupling lattice QCD for QCD phase diagram, Prog. Theor. Exp. Phys. 2014, 123D02 (2014); T. Ichihara, T.Z. Nakano, and A. Ohnishi, QCD phase diagram at strong coupling including auxiliary field fluctuations, Proc. Sci., LAT2013 (2014) 143 [arXiv:1311.5352]; A. Ohnishi, T. Ichihara, and T.Z. Nakano, Auxiliary field Monte-Carlo study of the QCD phase diagram at strong coupling, Proc. Sci., LAT2012 (2012) 088 [arXiv:1211.2282].

[34] A. Gocksch and M. Ogilvie, Finite temperature deconfinement and chiral symmetry restoration at strong coupling, Phys. Rev. D 31, 877 (1985).

[35] E. M. Ilgenfritz and J. Kripfganz, Dynamical fermions at nonzero chemical potential and temperature: Mean field approach, Z. Phys. C 29, 79 (1985).

[36] K. Fukushima, Relation between the Polyakov-loop and the chiral order parameter at strong coupling, Phys. Rev. D 68 , 045004 (2003).

[37] P. de Forcrand (private communication); For the temporal lattice extension $N_{t}=2$, the lattice bare coupling associated with the chiral phase transition $\left(\beta_{c}\right)$ is found to be 3.67 at the bare quark mass $m_{0}=0.025$ and 3.81 at $m_{0}=0.05$ for one species of staggered fermion.

[38] G. Boyd, J. Fingberg, F. Karsch, L. Karkkainen, and B. Petersson, Critical exponents of the chiral transition in strong coupling QCD, Nucl. Phys. B376, 199 (1992); R. V. Gavai, S. Gupta, A. Irbäck, F. Karsch, S. Meyer, B. Petersson, H. Satz, and H. W. Wyld (MT(c) Collaboration), Flavor degrees of freedom and the transition temperature in QCD, Phys. Lett. B 241, 567 (1990); S. A. Gottlieb, W. Liu, D. Toussaint, R. L. Renken, and R. L. Sugar, Chiral symmetry breaking in lattice QCD with two and four fermion flavors, Phys. Rev. D 35, 3972 (1987); A. D. Kennedy, J. Kuti, S. Meyer, and B. J. Pendleton, Where Is The Continuum In Lattice Quantum Chromodynamics?, Phys. Rev. Lett. 54, 87 (1985).

[39] J. Engels, R. Joswig, F. Karsch, E. Laermann, M. Lutgemeier, and B. Petersson, Thermodynamics of four 
flavor QCD with improved staggered fermions, Phys. Lett. B 396, 210 (1997).

[40] J. Polonyi and K. Szlachanyi, Phase transition from strong coupling expansion, Phys. Lett. 110B, 395 (1982); M. Gross, Deconfinement at strong coupling, Phys. Lett. 132B, 125 (1983); J. Bartholomew, D. Hochberg, P. H. Damgaard, and M. Gross, Effect of quarks on SU(N) deconfinement phase transitions, Phys. Lett. 133B, 218 (1983).

[41] P. H. Damgaard, N. Kawamoto, and K. Shigemoto, Strong coupling analysis of the chiral phase transition at finite temperature, Nucl. Phys. B264, 1 (1986).

[42] K. Fukushima, Chiral effective model with the Polyakovloop, Phys. Lett. B 591, 277 (2004).

[43] K. Fukushima, Phase diagrams in the three-flavor NambuJona-Lasinio model with the Polyakov-loop, Phys. Rev. D 77, 114028 (2008).

[44] In fact, the PNJL model is invented from the SC-LQCD [42].

[45] T. K. Herbst, J. M. Pawlowski, and B. J. Schaefer, The phase structure of the Polyakov-quark-meson model beyond mean field, Phys. Lett. B 696, 58 (2011); Phase structure and thermodynamics of QCD, Phys. Rev. D 88, 014007 (2013).

[46] T. Jolicoeur, H. Kluberg-Stern, M. Lev, A. Morel, and B. Petersson, The strong coupling expansion of lattice gauge theories with Susskind fermions, Nucl. Phys. B235, 455 (1984).

[47] T. Ichihara, A. Ohnishi, and K. Morita, Higher order net baryon number cumulants in the strong coupling lattice QCD, Proc. Sci., LATTICE 2015 (2016) 203.
[48] P. de Forcrand and O. Philipsen, The Chiral critical point of $\mathrm{N}(\mathrm{f})=3 \mathrm{QCD}$ at finite density to the order $(\mathrm{mu} / \mathrm{T})^{* *} 4, \mathrm{~J}$. High Energy Phys. 11 (2008) 012.

[49] R. D. Pisarski and F. Wilczek, Remarks on the chiral phase transition in chromodynamics, Phys. Rev. D 29, 338 (1984).

[50] I. Ichinose and K. Nagao, Lattice QCD with the overlap fermions at strong gauge coupling, Nucl. Phys. B577, 279 (2000); Lattice QCD with the overlap fermions at strong gauge coupling. 2, Nucl. Phys. B596, 231 (2001).

[51] X. L. Yu and X. Q. Luo, Phase structures of strong coupling lattice QCD with overlap fermions at finite temperature and chemical potential, Mod. Phys. Lett. A 22, 537 (2007).

[52] G. Bergner, J. Langelage, and O. Philipsen, Effective lattice Polyakov loop theory vs. full SU(3) Yang-Mills at finite temperature, J. High Energy Phys. 03 (2014) 039; J. Langelage and O. Philipsen, The pressure of strong coupling lattice QCD with heavy quarks, the hadron resonance gas model and the large N limit, J. High Energy Phys. 04 (2010) 055; The deconfinement transition of finite density QCD with heavy quarks from strong coupling series, J. High Energy Phys. 01 (2010) 089; J. Langelage, G. Munster, and O. Philipsen, Strong coupling expansion for finite temperature Yang-Mills theory in the confined phase, J. High Energy Phys. 07 (2008) 036.

[53] P. M. Lo, B. Friman, O. Kaczmarek, K. Redlich, and C. Sasaki, Probing deconfinement with Polyakov loop susceptibilities, Phys. Rev. D 88, 014506 (2013).

[54] H. Kluberg-Stern, A. Morel, and B. Petersson, Spectrum of lattice gauge theories with fermions from a 1/D expansion at strong coupling, Nucl. Phys. B215, 527 (1983). 